\title{
Langevin dynamics with a tilted periodic potential
}

\author{
Gioia Carinci ${ }^{(\mathrm{a})} \quad$ and $\quad$ Stephan Luckhaus ${ }^{(\mathrm{b})}$ \\ (a) University of Modena and Reggio Emilia, via G. Campi 213/b, 41125 Modena, Italy \\ e-mail: gioia.carinci@unimore.it
}

(b) University of Leipzig, Augustus Platz, 10-11, D-04109 Leipzig, Germany

e-mail: luckhaus@mis.mpg.de

\begin{abstract}
We study a Langevin equation for a particle moving in a periodic potential in the presence of viscosity $\gamma$ and subject to a further external field $\alpha$. For a suitable choice of the parameters $\alpha$ and $\gamma$ the related deterministic dynamics yields heteroclinic orbits. In such a regime, in absence of stochastic noise both confined and unbounded orbits coexist. We prove that, with the inclusion of an arbitrarly small noise only the confined orbits survive in a sub-exponential time scale.
\end{abstract}

\section{Introduction}

In many physical contexts e.g. the switching of magnetic domain walls (Barkhausen noise) or the motion of twin boundaries in crystals, one observes an intermittent dynamics of energy relaxation with "relaxation events" of random amplitude. This type of dynamics is commonly called Avalanche Dynamics (see e.g. 9, 10]). In order to give a possible explanation for this kind of mechanism, we need three ingredients: a rough interaction potential with many local energy barriers, and a small tilt; an "almost Hamiltonian" dynamics that approximately conserves the total energy, helping the system to overcome the next energy barrier once it passes the first one; finally a weak coupling to a heat bath. The simplest model one may consider in a two dimensional phase space taking into account all these factors is the Brownian motion in a tilted periodic potential. Let $x(t) \in \mathbb{R}$ be the coordinate of a one-dimensional Brownian particle moving in a periodic oscillating potential $V_{0}(x)$ (e. g. $\left.V_{0}(x)=\cos x\right)$ in the presence of viscosity $\gamma$ and subject to an additional constant external force $\alpha$. The dynamics for $x(t)$ is governed by the Langevin equation:

$$
\ddot{x}+\gamma \dot{x}+V_{0}^{\prime}(x)=\alpha+\epsilon \dot{w}(t)
$$

where $\dot{w}(t)$ is the white noise, and $\epsilon$ a small parameter. The model is not new, indeed it has been widely studied because of its well known applications to several classical areas of physics such as electronics (see e.g. [13, 14]) and solid state physics (superionic conductors and Josephson tunneling junction, see e.g. Section 11.1 of [11).

According to the values of the parameters and the initial conditions, the particle may escape in the direction of the force $\alpha$ or be trapped for a long time in one of the wells of the potential. Without any noise $(\epsilon=0)$, when $\alpha$ is large enough there are only "running solutions", i.e. unbounded solutions. When the force $\alpha$ is small enough and the friction parameter $\gamma$ is large enough, the particle finally reaches one of the minima of the potential. In this case there are only "locked solutions". For $\alpha$ and $\gamma$ small enough, both types of solution coexist. With the addition of the noise there are certainly transitions between the locked and the running state, due to large deviations effects and related to 
the problem of diffusion exit from a domain 3. These kind of transitions take place in the Kramer's escape time scale, i.e. the time required for a brownian particle to escape from a well in the presence of viscosity, that is of order of $\exp \left(\gamma / \epsilon^{2}\right)$ for $\epsilon^{2}<<\gamma<<1$ (see [5]). There is a wide literature concerning the diffusion along a periodic potential, mostly inspired by the Kramer's theory [5], that includes both numerical and theoretical studies (see e.g. [4, 8, 11, 12]). In the present paper we do not assume a large deviations point of view, since we are interested in a different time scale. In certain critical regimes, due to the instability of the dynamics, small stochastic perturbations may indeed affect the macroscopic behavior even in a faster time scale. This for instance turns out to be the case for the particular choice $\alpha=\alpha_{\gamma}$ yielding heteroclinic deterministic orbits (i.e. orbits connecting two consecutive maxima of the potential). In the present work we show that, by stochastically perturbing the system in the critical regime $\alpha=\alpha_{\gamma}$ that exibits both running and locked solutions, only the locked solutions survive in a subexponential time scale (and more precisely if one observes the system for times $\left.\ln \epsilon^{-1}<<T<<\exp \left(\gamma / \epsilon^{2}\right)\right)$. The introduction of an arbitrarly small noise has thus a macroscopic effect, since it reduces the bistability region even in this "fast time scale". The next case to consider is thus the region $\alpha>\alpha_{\gamma}$. In particular one may wonder whether there exists a transition regime from the fast to the slow time scale where only the Kramer's diffusion is observed.

\section{Deterministic orbits}

We denote by $V(x)$ the total potential taking into account also the linear term due to the external force, $V(x)=V_{0}(x)-\alpha x$. The equation of the motion 1.1 then becomes

$$
\ddot{x}+\gamma \dot{x}+V^{\prime}(x)=\epsilon \dot{w}(t)
$$

and the related first order system is

$$
\left\{\begin{array}{l}
\dot{x}=p \\
\dot{p}=-\gamma p-V^{\prime}(x)+\epsilon \dot{w} .
\end{array}\right.
$$

Consider the deterministic system related to $(1.3)$, i.e. the $\epsilon=0$ case,

$$
\left\{\begin{array}{l}
\dot{X}=P \\
\dot{P}=-\gamma P-V^{\prime}(X)
\end{array}\right.
$$

and sketch the phase diagram. In each period of the original potential $V_{0}(x)$ there are confined and escaping orbits. The picture changes according to $\alpha$ and $\gamma$.

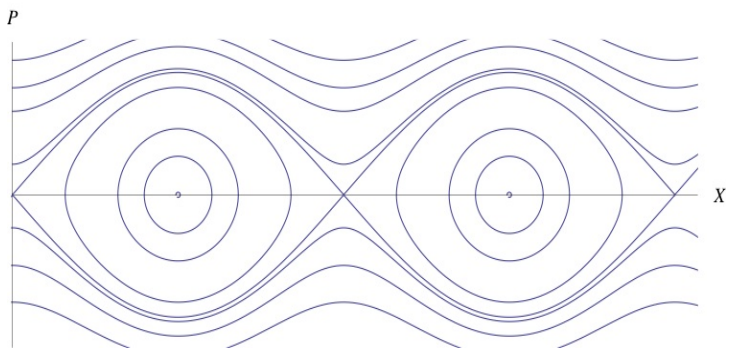

Figure 1: Phase diagram for $\alpha=\gamma=0$. The orbits are periodic.

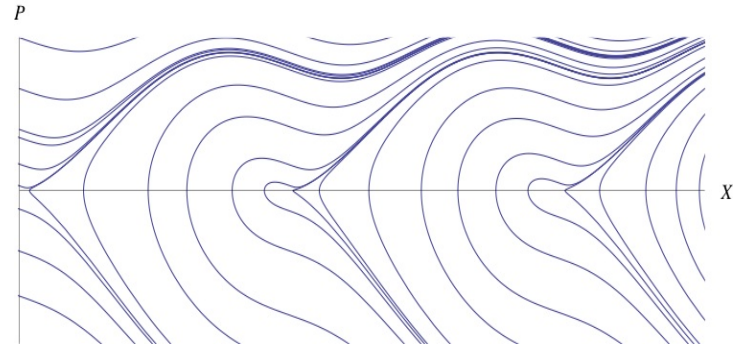

Figure 2: Phase diagram for $\alpha>1$. There are only running orbits.

In absence of viscosity and external force (i.e. for $\gamma=\alpha=0$ ), the orbits are periodic (see Figure 1). For positive $\gamma$ and $\alpha$ the dynamics loses its periodicity. The inclusion of the friction makes the system dissipative, the particles lose energy, and the confined orbits are attracted by the local minima of the potential, whereas the running solutions have an asymptotic effective velocity. 
Figures 2, 3 and 4 provide three possible phase diagrams. For $\alpha>1$ the total potential $V(x)$ does not have local minima and then there are only running solutions (see Figure 2).

When $\alpha<1$, there are also bounded solutions. For any $\gamma>0$, there exists a critical value $\alpha_{\gamma}>0$ such that, for $0<\alpha<\alpha_{\gamma}$, there are only confined orbits (see Figure 3). For $\alpha_{\gamma}<\alpha<1$ running and bounded orbits coexist (see Figure 4).

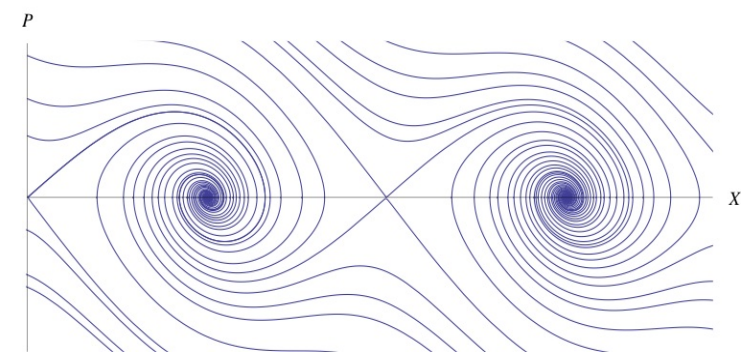

Figure 3: Phase diagram for $\gamma>0$ and $0<\alpha<\alpha_{\gamma}$. There are only confined orbits.

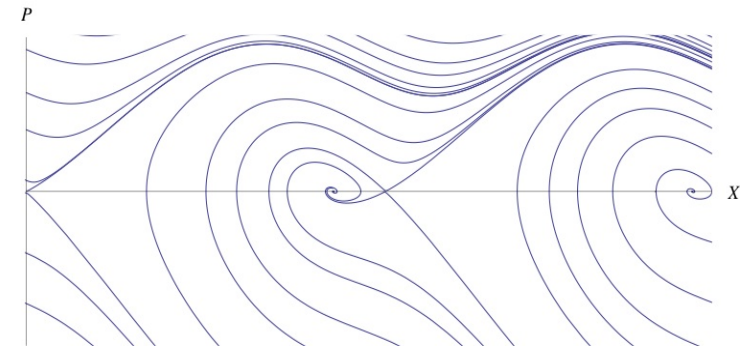

Figure 4: Phase diagram for $\gamma>0$ and $\alpha_{\gamma}<\alpha<1$. There are both running and and confined orbits.

The situation is well resumed in Figure 5 that shows the dependence on $\gamma$ of the critical value $\alpha_{\gamma}$. As we will see below, $\alpha_{\gamma}$ is of the order of $\gamma$ as $\gamma \rightarrow 0$.

For any $\alpha<1$ there exist critical orbits, i.e. orbits asymptotically converging to some saddle equilibria (corresponding to some local maxima of the potential) in the phase plane. The critical scaling $\alpha=\alpha_{\gamma}$ is the one that gives rise to heteroclinic orbits, i.e. orbits connecting two consecutive local maxima of the potential.

Each orbit can be piecewise expressed by a function in the phase plane that we will usually denote by $\wp(x)$. We say that $\wp(x)$ is an orbit of our dynamics if there exists a solution $(X(t), P(t))$ of $(1.4)$ and a suitable time interval $I \subseteq(0,+\infty)$ such that $\wp(X(t))=P(t)$ for any $t \in I . \wp(x)$ must verify the

$$
\frac{d \wp}{d x}(x)=-\gamma-\frac{V^{\prime}(x)}{\wp(x)}
$$

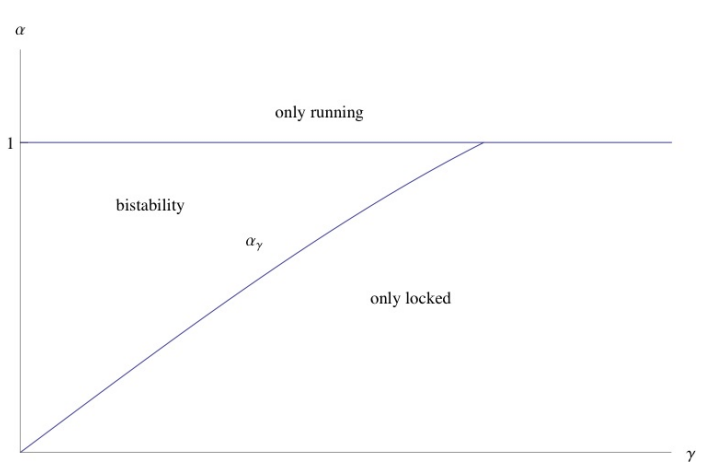

Figure 5: The picture shows the three different regimes in the plane $(\gamma, \alpha)$.

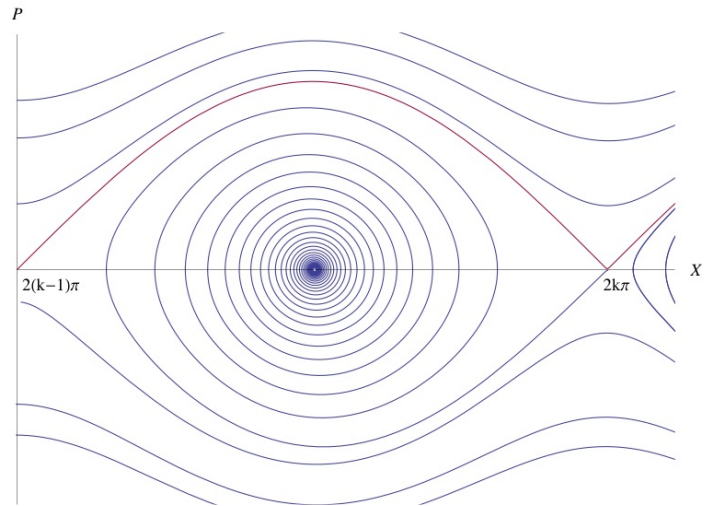

Figure 6: When $\alpha=\alpha_{\gamma}$ the heteroclinic orbit $\wp_{k}^{*}(x)$ (red line) separates the escaping and the confined solutions. 


\section{The problem}

In the present paper we are interested in the critical regime $\left(\alpha=\alpha_{\gamma}\right)$ dynamics in the small noise limit $(\epsilon \rightarrow 0)$. Thus from now on we fix $\gamma$ small enough and $\alpha=\alpha_{\gamma}$. We denote by $\wp_{k}^{*}(x)$ the $k$-th heteroclinic orbit, i.e. the orbit connecting the $k-1$-th maximum of the potential with the $k$-th one:

$$
\lim _{x \rightarrow 2(k-1) \pi^{+}} \wp_{k}^{*}(x)=\lim _{x \rightarrow 2 k \pi^{-}} \wp_{k}^{*}(x)=0
$$

see Figure 6

In this paper we are not concerned with large deviations. We investigate the problem in a subexponential time scale. Far from the critical orbits we expect that the noise does not macroscopically affect the deterministic dynamics in such a fast time scale. On the other hand, there may be a macroscopic perturbation of the deterministic dynamics in a neighbourhood of the heteroclinic orbits. Then we choose the initial value to lay on one of the heteroclinic orbits, i.e. we denote by $(x(t), p(t))$ the solution of the problem

$$
\begin{cases}\dot{x}=p & x(0)=-\pi \\ \dot{p}=-\gamma p-V^{\prime}(x)+\epsilon \dot{w} & p(0)=\wp_{0}^{*}(-\pi),\end{cases}
$$

and study the probability law of $(x(t), p(t))$ in the limit as $\epsilon \rightarrow 0$.

We show that, at each time, the probability for the particle, to get across the next well is $1 / 2$, in the limit as $\epsilon \rightarrow 0$. We prove, thus, that for fixed $\gamma$ small enough, the random variable associated to the number of wells passed by $(x(t), p(t))$ has, for vanishing $\epsilon$, a geometric distribution of parameter $1 / 2$. This implies, in particular, that the particle will finally be trapped in one of the wells for a long time, with probability 1 as $\epsilon \rightarrow 0$. The involved time scale, or more precisely, the velocity the particle travels before to be trapped in one of the wells is of order $\ln \epsilon$. With the inclusion of the noise, thus, the bistability between the locked and the running states is lost and only the locked state survives in this "fast time scale".

\section{Notation}

Before turning to the precise statement, we introduce some notations. We shall use

- $f(\delta)=o(g(\delta))$ as $\delta \rightarrow 0$ if $\lim _{\delta \rightarrow 0} f(\delta) / g(\delta)=0$;

- $f(\delta)=\mathcal{O}(g(\delta))$ as $\delta \rightarrow 0$ if there exists $c>0$ such that $|f(\delta)| \leq c|g(\delta)|$ for any sufficiently small $\delta$;

- $f(\delta)=\Theta(g(\delta))$ as $\delta \rightarrow 0$ if there exist $c_{1}, c_{2}>0$ such that $c_{1}|g(\delta)| \leq|f(\delta)| \leq c_{2}|g(\delta)|$ for any sufficiently small $\delta$;

- $X \sim \mu$ if the stochastic variable has probability law $\mu$.

\section{Basic parameters}

Although our results do not depend on the exact form of the potential, for the sake of simplicity we choose

$$
V(x)=\cos \left(x-x_{\alpha}\right)-\alpha\left(x-x_{\alpha}\right), \quad x_{\alpha}=\arcsin \alpha
$$

in such a way that $V(x)$ attains its local maxima at $x=2 k \pi$ and its local minima at $x=(2 k-1) \pi+2 x_{\alpha}$. Let us investigate how $\alpha_{\gamma}$ is related to $\gamma$. All the orbits $\wp_{k}^{*}(x)$ must verify the equation $(1.5)$, then

$$
\frac{1}{2} \frac{d}{d x}\left(\wp_{k}^{*}(x)\right)^{2}=-V^{\prime}(x)-\gamma \wp_{k}^{*}(x) .
$$


By integrating $(1.8)$ in $(2(k-1) \pi, 2 k \pi)$, and using the asympthotic conditions (1.6) we get

$$
2 \pi \frac{\alpha_{\gamma}}{\gamma}=\int_{2(k-1) \pi}^{2 k \pi} \wp_{k}^{*}(x) d x=\Theta(1) \quad \text { as } \quad \gamma \rightarrow 0
$$

thus $\alpha_{\gamma}=\Theta(\gamma)$ in the limit as $\gamma \rightarrow 0$.

We compute the asymptotic values of $d \wp_{k}^{*}(x) / d x$ for $x \rightarrow 2 k \pi^{ \pm}$. Let

$$
\beta=\beta_{\gamma}:=-V^{\prime \prime}(2 k \pi)=\sqrt{1-\alpha_{\gamma}^{2}}>0 \quad k \in \mathbb{Z},
$$

then, from 1.5 we obtain that the asympthotic slopes of the heteroclinic orbits:

$$
\lambda_{+}:=\frac{d}{d x} \wp_{k}^{*}\left(2(k-1) \pi^{+}\right) \quad \text { and } \quad \lambda_{-}:=\frac{d}{d x} \wp_{k}^{*}\left(2 k \pi^{-}\right)
$$

must satisfy the equation

$$
\lambda^{2}+\gamma \lambda-\beta=0
$$

then

$$
\lambda_{ \pm}=\lambda_{ \pm}^{\gamma}=\frac{-\gamma \pm \sqrt{\gamma^{2}+4 \beta_{\gamma}}}{2}
$$

notice that $\lim _{\gamma \rightarrow 0} \beta_{\gamma}=1$ and $\lim _{\gamma \rightarrow 0} \lambda_{\gamma}^{ \pm}= \pm 1$. We define, moreover, the parameter

$$
\theta=\theta_{\gamma}:=\frac{\left|\lambda_{-}\right|}{\lambda_{+}}-1=\frac{2 \gamma}{\sqrt{\gamma^{2}+4 \beta}-\gamma}
$$

then $\theta_{\gamma}=\Theta(\gamma)$ as $\gamma \rightarrow 0$ with $\lim _{\gamma \rightarrow 0} \theta_{\gamma} / \gamma=1$.

\section{Critical region dynamics}

In a neighborhood of the criticalities, i.e. when $x(t)$ approaches $2 k \pi, V^{\prime}(x(t))$ is well approximated by $-\beta(x(t)-2 k \pi)$, since $V^{\prime}(2 k \pi)=0$. Hence the dynamics can be approximated by the linear system

$$
\left\{\begin{array}{l}
\dot{x}=p \\
\dot{p}=-\gamma p+\beta(x-2 k \pi)+\epsilon \dot{w}
\end{array}\right.
$$

A convenient choice of variables is given by

$$
\left\{\begin{array}{l}
z_{k}(t):=p(t)-\lambda_{-}(x(t)-2 k \pi) \\
v_{k}(t):=p(t)-\lambda_{+}(x(t)-2 k \pi)
\end{array}\right.
$$

with $\lambda_{ \pm}$as defined in 1.11 . Since in these variables the linearized system 1.13 becomes

$$
\left\{\begin{array}{l}
\dot{z}=\lambda_{+} z+\epsilon \dot{w} \\
\dot{v}=\lambda_{-} v+\epsilon \dot{w}
\end{array}\right.
$$

where the equations are coupled only per the stochastic term.

Since $\wp_{k}^{*}(2 k \pi)=0$, by 1.10 , for $|x(t)-2 k \pi|$ small enough we have

$$
z_{k}(t)=p(t)-\wp_{k}^{*}(x(t))+\mathcal{O}\left((x(t)-2 k \pi)^{2}\right) \quad \text { for } \quad x(t)<2 k \pi
$$

and

$$
v_{k}(t)=p(t)-\wp_{k+1}^{*}(x(t))+\mathcal{O}\left((x(t)-2 k \pi)^{2}\right) \quad \text { for } \quad x(t)>2 k \pi .
$$

The variable $\left|z_{k}(t)\right|$ can, thus, be thought of as a measure of the distance, in the phase plane, from the $k$-th heteroclinic orbit $\wp_{k}^{*}(\cdot)$ just before the $k$-th criticality. Equivalently $\left|v_{k}(t)\right|$ is a measure of the distance from the $k+1$-th heteroclinic orbit $\wp_{k+1}^{*}(\cdot)$ just after the $k$-th criticality. 


\section{Stopping times}

Far from criticalities the dynamics is stable and we expect the distribution of our process to be quite concentred in a neighborhood of some deterministic paths (see the deterministic system (1.4)).

One of the main technicalities we run into in the proof of our result is the choice of the length of the critical interval. It is clear that the linear system 1.13 is a good approximation of our dynamics as long as $x(t)$ is close enough to the $k$-th criticality $2 k \pi$. This provides an upper bound on the critical interval length. On the other hand, we need a good localization of the process at the beginning of the critical interval. We want the process to be still quite concetrated and not to diffuse too much. This clearly requires a lower bound on the length of the critical interval.

We denote by $\eta_{\epsilon}$ the order of magnitude of the length of the critical interval. Then, in order to make valid our approximations we need to impose the following condition on $\eta_{\epsilon}$ :

$$
\eta_{\epsilon}=\epsilon^{\nu} \quad \text { with } \quad \frac{(1+\theta)^{2}}{3+2 \theta}<\nu<\frac{1}{2}
$$

and $\theta$ as in 1.12 .

We define two sequences of stopping times. We call $S_{k}$ the first time the process gets into the $k$-th critical interval and by $T_{k+1}$ the first exit time from the $k$-th critical interval. The rigorous definition is given as follows

$$
\begin{aligned}
& S_{k}:=\inf \left\{t \geq 0: v_{k}(t) \leq \eta_{\epsilon}\right\}, \quad k \geq 0 \\
& T_{k}:=\inf \left\{t \geq S_{k-1}:\left|z_{k-1}(t)\right| \geq \eta_{\epsilon}\right\}, \quad k \geq 1
\end{aligned}
$$

We will see that, under the condition (1.18), both $\left|z_{k}\left(S_{k}\right)\right|$ and $\left|v_{k}\left(T_{k+1}\right)\right|$ are $o\left(\eta_{\epsilon}\right)$ with large probability, then

$$
2 k \pi-x\left(S_{k}\right)=\Theta\left(\eta_{\epsilon}\right) \quad \text { and } \quad\left|x\left(T_{k+1}\right)-2 k \pi\right|=\Theta\left(\eta_{\epsilon}\right)
$$

with large probability, since $v_{k}\left(S_{k}\right)=\left|z_{k}\left(T_{k+1}\right)\right|=\eta_{\epsilon}$ and $x(\cdot)-2 k \pi=\left[z_{k}(\cdot)-v_{k}(\cdot)\right] /\left(\lambda_{+}-\lambda_{-}\right)$.

At time $S_{k}$ the fundamental variable is $z_{k}\left(S_{k}\right)$, since, as we showed before, $\left|z_{k}\left(S_{k}\right)\right|$ measures the distance from the $k$-th deterministic heteroclinic path before the criticality.

At time $T_{k+1}$ the fundamental variable is $v_{k}\left(T_{k}\right)$ since $\left|v_{k}\left(T_{k+1}\right)\right|$ is a measure of the distance from the $k+1$-th heteroclinic path, or, if the well has not been crossed, a measure of the distance from a suitable locked deterministic path.

$\operatorname{sign}\left(z_{k}\left(T_{k+1}\right)\right)$ establishes whether the solution has crossed the $k$-th criticality or not. If $z_{k}\left(T_{k+1}\right)>0$ the $k$-th criticality has been passed by, if $z_{k}\left(T_{k+1}\right)<0$ the solution has been trapped in the $k-1$-th well.

\section{The Result}

We investigate the probability law of the number of wells crossed by $(x(t), p(t))$, thus we define the random variable

$$
\mathcal{N}:=\inf \left\{k \geq 0: z_{k}\left(T_{k+1}\right)<0\right\} \in \mathbb{N} \cup\{0\}
$$

We look at the process stopped at time $T_{\mathcal{N}+1},\left(x\left(t \wedge T_{\mathcal{N}+1}\right), p\left(t \wedge T_{\mathcal{N}+1}\right)\right)$. All the processes labeled by $k$ defined in the previous Sections (e.g. $\left.\left(z_{k}(t), v_{k}(t)\right)\right)$ are well defined for $k \leq \mathcal{N}$. $S_{k}$ is defined for $k \leq \mathcal{N}$ and $T_{k}$ for $k \leq \mathcal{N}+1$.

We denote by $\mathbf{P}$ the probability law of $\left(x\left(t \wedge T_{\mathcal{N}+1}\right), p\left(t \wedge T_{\mathcal{N}+1}\right)\right)$, the main result is given by the following Theorem.

Theorem 1.1. There exists $c>0$ such that

$$
\left(\frac{1}{2}-c \epsilon^{\theta}\right)^{k+1} \leq \mathbf{P}\{\mathcal{N}=k\} \leq\left(\frac{1}{2}+c \epsilon^{\theta}\right)^{k+1}
$$

for any $k \in \mathbb{N} \cup\{0\}$ and $\epsilon$ small enough. 
Corollary 1.2. From Theorem 1.1 it follows that the r.v. $\mathcal{N}+1$ has, in the limit as $\epsilon \rightarrow 0$, a geometric distribution of parameter $1 / 2$, i.e.

$$
\lim _{\epsilon \rightarrow 0} \mathbf{P}\{\mathcal{N}=k\}=\frac{1}{2^{k+1}}
$$

This implies, in particular, that the process crosses a finite number of wells:

$$
\lim _{\epsilon \rightarrow 0} \mathbf{P}\{\mathcal{N}<\infty\}=1
$$

$\mathcal{N}$ is the number of wells crossed by the process, in the sense that the first well our process is trapped is the $\mathcal{N}$-th one, i.e. the one where the potential has a local minimum at $(2 \mathcal{N}-1) \pi+2 x_{\alpha}$.

Proposition 1.3. There exists $c>0$ such that

$$
\lim _{\epsilon \rightarrow 0} \mathbf{P}\left\{x\left(T_{\mathcal{N}+1}\right) \leq 2 \mathcal{N} \pi-\frac{\eta_{\epsilon}}{\left(\lambda_{+}-\lambda_{-}\right)}(1-o(1)), p\left(T_{\mathcal{N}+1}\right)<-\frac{\lambda_{+} \eta_{\epsilon}}{\left(\lambda_{+}-\lambda_{-}\right)}(1-o(1))\right\}=1 .
$$

Once $(x(t), p(t))$ is trapped in the $\mathcal{N}$-well in the sense of Proposition 1.3 , we expect that it remains confined in it for a long time. It is quite reasonable to think that, due to the stochastic fluctuations, the process will leave the well in a exponential time scale (order of $e^{\epsilon^{-1}}$ ). This is not a problem we are concerned with, since, at this level, we are looking at a faster time scale, indeed, as we will see, the time required to get across a criticality is of order $\ln \left(\epsilon^{-1}\right)$. We say, thus, that the process is "confined in the $\mathcal{N}$-th well" in the sense that $x(t) \in(2(\mathcal{N}-1) \pi, 2 \mathcal{N} \pi)$, after $T_{\mathcal{N}+1}$, for a time that is very long if compared with $T_{\mathcal{N}+1}$ itself. For this reason it is quite reasonable to stop the process at time $T_{\mathcal{N}+1}$.

\section{Definitions}

The tecniques used to prove our result are based on a pathwise analysis of the stochastic trajectories, (to this end see e. g. [1). We perform two different linearizations of the nonlinear equation (1.3). In the neighborhoods of the criticalities we use the linearization in the system 1.13 that is solved by a gaussian process. In the stable region $(x(t), p(t))$ is expected to track a suitable deterministic trajectory, thus linearization is done around a given deterministic solution. Then we study the behavior of the approximating trajectories and use comparison techniques to get from them a control on the original process.

\section{Dynamics near the criticalities}

For $S_{k}$ as in 1.19 , the process $\left(z_{k}(t), v_{k}(t)\right)$ defined in 1.14 satisfies the problem

$$
\begin{cases}\dot{z}:=\lambda_{+} z+\psi_{k}(x(t))+\epsilon \dot{w} & z_{k}\left(S_{k}\right)=p\left(S_{k}\right)-\lambda_{-}\left(x\left(S_{k}\right)-2 k \pi\right) \\ \dot{v}:=\lambda_{-} v+\psi_{k}(x(t))+\epsilon \dot{w} & v_{k}\left(S_{k}\right)=\eta_{\epsilon}\end{cases}
$$

with

$$
\psi_{k}(x)=-V^{\prime}(x)-\beta(x-2 k \pi)=\mathcal{O}\left((x-2 k \pi)^{2}\right) \quad \text { as } \quad x \rightarrow 2 k \pi
$$

We denote by $\left(\bar{z}_{k}(t), \bar{v}_{k}(t)\right)$ the solution of the related linear problem 1.15) starting from the same point: $\left(z_{k}\left(S_{k}\right), \eta_{\epsilon}\right)$. Thus, for $t \geq S_{k}$,

$$
\bar{z}_{k}(t)=z_{k}\left(S_{k}\right) e^{\lambda_{+}\left(t-S_{k}\right)}+\epsilon e^{\lambda_{+} t} \int_{S_{k}}^{t} e^{-\lambda_{+} s} d w_{s}
$$

and

$$
\bar{v}_{k}(t)=\eta_{\epsilon} e^{\lambda_{-}\left(t-S_{k}\right)}+\epsilon e^{\lambda_{-} t} \int_{S_{k}}^{t} e^{-\lambda_{-} s} d w_{s} .
$$


We define the errors

$$
\mathcal{V}_{k}(t):=v_{k}(t)-\bar{v}_{k}(t) \quad \text { and } \quad \mathcal{Z}_{k}(t):=z_{k}(t)-\bar{z}_{k}(t)
$$

thus

$$
\mathcal{V}_{k}(t)=e^{\lambda_{-} t} \int_{S_{k}}^{t} e^{-\lambda_{-} s} \psi_{k}(x(s)) d s \quad \text { and } \quad \mathcal{Z}_{k}(t)=e^{\lambda_{+} t} \int_{S_{k}}^{t} e^{-\lambda_{+} s} \psi_{k}(x(s)) d s
$$

\section{Dynamics far from criticalities}

Suppose that $T_{k}<\infty$ and that the solution has crossed the $k$-1-th criticality, then we denote by $\left(X_{k}(t), P_{k}(t)\right), t \geq T_{k}$ the deterministic path starting in $T_{k}$ from $\left(x\left(T_{k}\right), p\left(T_{k}\right)\right)$, i.e. the solution of the problem

$$
\begin{cases}\dot{X}=P & X_{k}\left(T_{k}\right)=x\left(T_{k}\right) \\ \dot{P}=-\gamma P-V^{\prime}(X) & P_{k}\left(T_{k}\right)=p\left(T_{k}\right)\end{cases}
$$

then we expect that, as long as $x(t)$ is far enough from the criticalities, the dynamics is concentrated in a neighborhood of $\left(X_{k}(t), P_{k}(t)\right)$. We denote by

$$
y_{k}(t):=x(t)-X_{k}(t) \quad \text { and } \quad q_{k}(t):=p(t)-P_{k}(t)
$$

thus $\left(y_{k}(t), q_{k}(t)\right)$ is solution of

$$
\begin{cases}\dot{y}=q & y_{k}\left(T_{k}\right)=0 \\ \dot{q}=-\gamma q-V^{\prime \prime}\left(X_{t}\right) y+\varphi\left(X_{k}(t), y\right)+\epsilon \dot{w} & q_{k}\left(T_{k}\right)=0\end{cases}
$$

with

$$
\varphi(X, y):=V^{\prime \prime}(X) y-\left[V^{\prime}(X+y)-V^{\prime}(X)\right]=\mathcal{O}\left(y^{2}\right), \quad \text { as } \quad|y| \rightarrow 0
$$

Assuming, for the moment, that it is possible, we define $\wp_{k}(x)$ as the curve on the phase plane associated to $\left(X_{k}(t), P_{k}(t)\right), t \in\left[T_{k}, S_{k}\right]$, i.e. the function such that $\wp_{k}\left(X_{k}(t)\right)=P_{k}(t)$ for any $t \in$ $\left[T_{k}, S_{k}\right]$. We know from 1.5 that $\wp_{k}(x)$ verifies the equation

$$
\wp^{\prime \prime}(x) \wp(x)=-\left(\wp^{\prime}(x)\right)^{2}-\gamma \wp^{\prime}(x)-V^{\prime \prime}(x),
$$

that is obtained by deriving (1.5). Let us define the function

$$
\omega_{k}(t):=\frac{d}{d x} \wp_{k}\left(X_{k}(t)\right)
$$

then, by using (2.8), we deduce that $\omega_{k}(t)$ verifies the equation

$$
\dot{\omega}=-\omega^{2}-\gamma \omega-V^{\prime \prime}\left(X_{k}(t)\right) .
$$

It turns out to be particularly convenient to pass to the variables $\left(y_{k}(t), r_{k}(t)\right)$, with

$$
r_{k}(t):=q_{k}(t)-\omega_{k}(t) y_{k}(t) \quad t \geq T_{k} .
$$

thus $\left(y_{k}(t), r_{k}(t)\right)$ is solution of the problem

$$
\begin{cases}\dot{y}=r+\omega_{k}(t) y & y_{k}\left(T_{k}\right)=0 \\ \dot{r}=-\left(\gamma+\omega_{k}(t)\right) r+\varphi\left(X_{k}(t), y_{k}(t)\right)+\epsilon \dot{w} & r_{k}\left(T_{k}\right)=0\end{cases}
$$

with $\varphi(X, y)$ as above. We denote by $\left(\bar{y}_{k}(t), \bar{r}_{k}(t)\right)$ the solution of the associated linear problem:

$$
\begin{cases}\dot{y}=r+\omega_{k}(t) y & \bar{y}_{k}\left(T_{k}\right)=0 \\ \dot{r}=-\left(\gamma+\omega_{k}(t)\right) r+\epsilon \dot{w} & \bar{r}_{k}\left(T_{k}\right)=0\end{cases}
$$


The convenience of this change of variables lies in the fact that the second equation in $(2.13)$ can be solved autonomously, and then $\bar{r}_{k}(t)$ can be made explicit as a function of $\omega_{k}(t)$. We have

$$
\bar{r}_{k}(t)=\epsilon \int_{T_{k}}^{t} e^{-\int_{s}^{t}\left(\omega_{k}\left(s^{\prime}\right)+\gamma\right) d s^{\prime}} d w_{s}
$$

Through 2.14 an explicit formula can be found also for $\bar{y}_{k}(t)$ as a function of $\omega_{k}(t)$,

$$
\bar{y}_{k}(t)=\int_{T_{k}}^{t} \bar{r}_{k}(s) e^{\int_{s}^{t} \omega_{k}\left(s^{\prime}\right) d s^{\prime}} d s=\epsilon \int_{T_{k}}^{t}\left(\int_{s}^{t} e^{-\int_{s}^{s^{\prime}}\left(2 \omega_{k}\left(s^{\prime \prime}\right)+\gamma\right) d s^{\prime \prime}} d s^{\prime}\right) d w_{s} .
$$

Finally we define the errors

$$
\mathcal{Y}_{k}(t):=y_{k}(t)-\bar{y}_{k}(t) \quad \text { and } \quad \mathcal{R}_{k}(t):=r_{k}(t)-\bar{r}_{k}(t)
$$

then

$$
\mathcal{Y}_{k}(t)=\int_{T_{k}}^{t} e^{\int_{s}^{t} \omega_{k}\left(s^{\prime \prime}\right) d s^{\prime \prime}}\left(\int_{s}^{t} e^{-\int_{s}^{s^{\prime}}\left(2 \omega_{k}\left(s^{\prime \prime}\right)+\gamma\right) d s^{\prime \prime}} d s^{\prime}\right) \varphi\left(X_{k}(s), y_{k}(s)\right) d s
$$

and

$$
\mathcal{R}_{k}(t)=\int_{T_{k}}^{t} e^{-\int_{s}^{t}\left(\omega_{k}\left(s^{\prime}\right)+\gamma\right) d s^{\prime}} \varphi\left(X_{k}(s), y_{k}(s)\right) d s
$$

\section{Remarks}

As long as $\left|y_{k}(t)\right|$ is small enough, thus far from the criticalities,

$$
r_{k}(t)=p(t)-\wp_{k}\left(X_{k}(t)\right)-\frac{d}{d x} \wp_{k}\left(X_{k}(t)\right) y_{k}(t)=p(t)-\wp_{k}(x(t))+\mathcal{O}\left(y_{k}^{2}(t)\right)
$$

and, since we expect $\wp_{k}(x)$ to be close enough to the $k$-th heteroclinic orbit $\wp_{k}^{*}(x)$, we have

$$
r_{k}(t) \simeq p(t)-\wp_{k}^{*}(x(t))
$$

The choice of the variables $\left(y_{k}(t), r_{k}(t)\right)$, thus, turns out to be particularly advantageous. Indeed, with a good choice of the parameter $\eta_{\epsilon}, r_{k}(t)$ is not far from $z_{k}(t)$ just before the criticality and from $v_{k}(t)$ just after it, i.e.

$$
r_{k}\left(S_{k}\right) \simeq z_{k}\left(S_{k}\right) \text { and } \quad r_{k+1}\left(T_{k+1}\right) \simeq v_{k}\left(T_{k+1}\right)
$$

as it is clear from 1.16), 1.17) and 2.18). Under this change of variables, the dynamics far from criticalities becomes "almost unidimensional". Getting in the $k$-th criticality we just need, as input, the distribution of $z_{k}\left(S_{k}\right)$ that is provided, unless small errors, by $r\left(S_{k}\right)$. Departing from the $k$-th criticality we get as output the distribution of $v_{k}\left(T_{k+1}\right) \mid\left\{z_{k}\left(T_{k+1}\right)>0\right\}$ that provides the approximated value of $r_{k+1}\left(T_{k+1}\right)$. Away from the criticalities, the fundamental variable is thus $r_{k}(t)$ and we can neglect to carefully analyse the behavior of $y_{k}(t)$. Since the linearization of $r_{k}(t)$ has a quite simple form as a function of $\omega_{k}(t)=\wp_{k}\left(X_{k}(t)\right)$ (see 2.14) ) everything can be computed with a good accuracy.

We introduce some of the parameters involved. Assuming that the " $k-1$-th criticality has been crossed", we will prove in Section 3 that for any $k, \bar{z}_{k}\left(S_{k}\right) \mid S_{k}, T_{k}$ is a Gaussian r.v. of standard deviation $\Theta\left(\sigma_{\epsilon}\right)$ and expected value that is a $\mathcal{O}\left(\tilde{\sigma}_{\epsilon}\right)$, with

$$
\sigma_{\epsilon}:=\epsilon \eta_{\epsilon}^{-\frac{1}{1+\theta}}=\epsilon^{1-\frac{\nu}{\theta+1}} \quad \text { and } \quad \tilde{\sigma}_{\epsilon}:=\bar{\sigma}_{\epsilon} \eta_{\epsilon}^{\frac{\gamma}{\beta} \sqrt{\gamma^{2}+4 \beta}}=\sigma_{\epsilon} \epsilon^{\theta} .
$$


On the other hand, we will see that $\bar{v}_{k}\left(T_{k+1}\right) \mid S_{k}$ in both cases (the $k$-th criticality has been/not been crossed) is, for any $k$, a Gaussian r.v. of standard deviation $\Theta(\epsilon)$ and expected value that is a $\mathcal{O}\left(\bar{\sigma}_{\epsilon}\right)$ with

$$
\bar{\sigma}_{\epsilon}:=\sigma_{\epsilon}^{1+\theta} \eta_{\epsilon}^{-\theta}=\sigma_{\epsilon} \epsilon^{\left(1-\frac{2+\theta}{1+\theta} \nu\right) \theta}
$$

It is easy to check that, under the condition (1.18) the following asympthotic relations hold that will be fundamental in our proof

$$
\sigma_{\epsilon}=o(1), \quad \bar{\sigma}_{\epsilon}=o\left(\sigma_{\epsilon}\right) \quad \text { and } \quad \tilde{\sigma}_{\epsilon}=o\left(\bar{\sigma}_{\epsilon}\right) \quad \text { as } \quad \epsilon \rightarrow 0
$$

and

$$
\epsilon=o\left(\eta_{\epsilon}^{2}\right), \quad \sigma_{\epsilon}=o\left(\eta_{\epsilon}\right) \quad \text { and } \quad \eta_{\epsilon}^{2}=o\left(\tilde{\sigma}_{\epsilon}\right) \quad \text { as } \quad \epsilon \rightarrow 0
$$

The stable region linearization $(1.13)$ is helpful as long as the dispersion around the heteroclinic solution is smaller than the distance $2 k \pi-x(t)$ itself, then we impose $\left|\bar{z}_{k}\left(S_{k}\right)\right|<2 k \pi-x\left(S_{k}\right)=\Theta\left(\eta_{\epsilon}\right)$, this provides the condition $\sigma_{\epsilon}=o\left(\eta_{\epsilon}\right)$ and then the upper bound in (1.18).

On the other hand, the errors due to the linearization in the critical interval $(1.13)$ are of order $(x(t)-2 k \pi)^{2}=\Theta\left(\eta_{\epsilon}^{2}\right)$. We want such an error to be small compared with the minimum distance from the heteroclinic solution, then we impose $(x(t)-2 k \pi)^{2}<\left|\bar{v}_{k}(t)\right| \wedge\left|\bar{z}_{k}(t)\right|$ for $t \in\left[S_{k}, T_{k+1}\right]$. This yields $\eta_{\epsilon}^{2}=o\left(\tilde{\sigma}_{\epsilon}\right)$ and thus the lower bound in 1.18$)$.

\section{Proof of the main result}

In this Section we prove Theorem 1.1 assuming the estimates of the errors due to the linearizations obtained in Section 6 and the estimates of the variances computed in Section 5 .

Lemma 3.1. For any fixed $k, t \geq 0$,

$$
\bar{v}_{k}\left(S_{k}+t\right) \sim \operatorname{Gauss}\left(\mu_{v}(t), \sigma_{v}(t)\right)
$$

with

$$
\mu_{v}(t)=\eta_{\epsilon} e^{\lambda_{-} t}, \quad \text { and } \quad \sigma_{v}^{2}(t)=\frac{\epsilon^{2}}{2\left|\lambda_{-}\right|}\left(1-e^{2 \lambda_{-} t}\right)
$$

$\bar{v}_{k}\left(S_{k}+t\right), t \geq 0$ is independent of $\sigma\left(S_{k}\right)$.

Proof. Recall the definition of $\bar{v}_{k}(t)$ in $(2.2)$ then it is clear that $\bar{v}_{k}\left(S_{k}+t\right) \mid S_{k}, t \geq 0$ has a Gaussian probability law whose average and variance are given by

$$
\mathbf{E}\left[\bar{v}_{k}\left(S_{k}+t\right) \mid S_{k}\right]=\eta_{\epsilon} e^{\lambda_{-} t}
$$

and

$$
\begin{array}{r}
\operatorname{var}\left[\bar{v}_{k}\left(S_{k}+t\right) \mid S_{k}\right]=\mathbf{E}\left[\left(\bar{v}_{k}\left(S_{k}+t\right)-\mathbf{E}\left[\bar{v}_{k}\left(S_{k}+t\right) \mid S_{k}\right]\right)^{2} \mid S_{k}\right] \\
=\epsilon^{2} \mathbf{E}\left[e^{2 \lambda_{-}\left(S_{k}+t\right)}\left(\int_{S_{k}}^{S_{k}+t} e^{-\lambda_{-} s} d w_{s}\right)^{2} \mid S_{k}\right] \\
=\epsilon^{2} \mathbf{E}\left[e^{2 \lambda_{-}\left(S_{k}+t\right)} \int_{S_{k}}^{S_{k}+t} e^{-2 \lambda_{-} s} d s\right]=\frac{\epsilon^{2}}{2\left|\lambda_{-}\right|}\left(1-e^{2 \lambda_{-} t}\right),
\end{array}
$$

then follows the result. 
Lemma 3.2. We have

$$
\bar{z}_{k}\left(S_{k}+t\right)-\bar{z}_{k}\left(S_{k}\right) e^{\lambda_{+} t} \sim \operatorname{Gauss}\left(0, \sigma_{z}(t)\right), \quad \sigma_{z}^{2}(t):=\frac{\epsilon^{2}}{2 \lambda_{+}}\left(e^{2 \lambda_{+} t}-1\right) .
$$

Proof. See the proof of Lemma 3.1 .

Let us define the process

$$
\begin{array}{r}
\hat{z}_{k}(t):=\hat{z}_{k}\left(S_{k}\right) e^{\lambda_{+}\left(t-S_{k}\right)}+\epsilon e^{\lambda_{+} t} \int_{S_{k}}^{t} e^{-\lambda_{+} s} d w_{s}, \\
\text { with } \quad \hat{z}_{k}\left(S_{k}\right):=P_{k}\left(S_{k}\right)-\wp_{k}^{*}\left(X_{k}\left(S_{k}\right)\right)+\bar{r}_{k}\left(S_{k}\right)
\end{array}
$$

and

$$
\mathcal{E}_{k}(t):=\mathcal{Z}_{k}(t)+\left(z_{k}\left(S_{k}\right)-\hat{z}_{k}\left(S_{k}\right)\right) e^{\lambda_{+}\left(t-S_{k}\right)}
$$

then, by 2.1), 2.3) and (3.4)

$$
\left[z_{k}(t) \mid z_{k-1}\left(T_{k}\right)>0\right] \sim \hat{z}_{k}(t)+\mathcal{E}_{k}(t), \quad t \geq S_{k} .
$$

Lemma 3.3. For $t \geq 0$,

$$
\hat{z}_{k}\left(S_{k}+t\right) \mid \hat{z}_{k}\left(S_{k}\right)=z \sim \operatorname{Gauss}\left(\mu_{z}(z, t), \sigma_{z}(t)\right)
$$

with

$$
\mu_{z}(z, t)=z e^{\lambda_{+}{ }^{t},} \quad \sigma_{z}^{2}(t) \quad \text { as in } 3.2 \text {. }
$$

Proof. See the proof of Lemma 3.1 .

Lemma 3.4. We have

$$
\hat{z}_{k}\left(S_{k}\right) \mid S_{k}, T_{k} \sim \operatorname{Gauss}\left(P_{k}\left(S_{k}\right)-\wp_{k}^{*}\left(X_{k}\left(S_{k}\right)\right), \sigma_{r}^{2}\left(T_{k}, S_{k}\right)\right)
$$

with

$$
\sigma_{r}^{2}\left(T_{k}, S_{k}\right):=\epsilon^{2} \int_{T_{k}}^{S_{k}} e^{-2 \int_{s}^{t}\left(\omega_{k}\left(s^{\prime}\right)+\gamma\right) d s^{\prime}} d s,
$$

$\omega_{k}(t)$ as in 2.9$)$.

Proof. From the definitions of $\hat{z}_{k}\left(S_{k}\right)$ and $\bar{r}_{k}(t)$ in (3.3) and (2.14), we have

$$
\hat{z}_{k}\left(S_{k}\right)=P_{k}\left(S_{k}\right)-\wp_{k}^{*}\left(X_{k}\left(S_{k}\right)\right)+\epsilon \int_{T_{k}}^{S_{k}} e^{-\int_{s}^{S_{k}}\left(\omega_{k}\left(s^{\prime}\right)+\gamma\right) d s^{\prime}} d w_{s}
$$

thus (3.7) follows. 
Lemma 3.5. We have

$$
\hat{z}_{k}\left(S_{k}+t\right) \mid S_{k}, T_{k} \sim \operatorname{Gauss}\left(\mu_{z, k}(t), \sigma_{z, k}(t)\right)
$$

with

$$
\mu_{z, k}(t)=e^{\lambda_{+}}{ }^{t}\left(P_{k}\left(S_{k}\right)-\wp_{k}^{*}\left(X_{k}\left(S_{k}\right)\right)\right)
$$

and

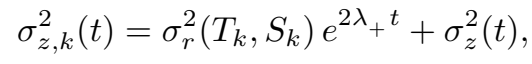

$\sigma_{z}^{2}(t)$ as in 3.6 .

Proof. From the definition of $\hat{z}_{k}(t)$ and by Lemma 3.4 we know that $\hat{z}_{k}\left(S_{k}+t\right)$ is the sum of two processes that, given $S_{k}, T_{k}$ have a Gaussian probability law, thus also $\hat{z}_{k}\left(S_{k}+t\right) \mid S_{k}, T_{k}$ has a Gaussian law. We have

$$
\mathbf{E}\left[\hat{z}_{k}\left(S_{k}+t\right) \mid S_{k}, T_{k}\right]=\mathbf{E}\left[\hat{z}_{k}\left(S_{k}\right) \mid S_{k}, T_{k}\right] e^{\lambda_{+} t}
$$

that yields (3.9). By the Ito's formula

$$
\hat{z}_{k}^{2}\left(S_{k}+t\right)=\hat{z}_{k}^{2}\left(S_{k}\right)+\int_{S_{k}}^{S_{k}+t}\left(2 \lambda_{+} \hat{z}_{k}^{2}(s)+\epsilon^{2}\right) d s+2 \epsilon \int_{S_{k}}^{S_{k}+t} \hat{z}_{k}(s) d w_{s}
$$

thus the function $f(t):=\mathbf{E}\left[\hat{z}_{k}^{2}\left(S_{k}+t\right) \mid S_{k}, T_{k}\right]$ satisfies the equation

$$
\frac{d}{d t} f(t)=2 \lambda_{+} f(t)+\epsilon^{2}
$$

then

$$
\begin{array}{r}
\operatorname{var}\left[\hat{z}_{k}\left(S_{k}+t\right) \mid S_{k}, T_{k}\right]=\mathbf{E}\left[\hat{z}_{k}^{2}\left(S_{k}+t\right) \mid S_{k}, T_{k}\right] \\
\quad=\mathbf{E}\left[\hat{z}_{k}^{2}\left(S_{k}\right) \mid S_{k}, T_{k}\right] e^{2 \lambda_{+} t}+\frac{\epsilon^{2}}{2 \lambda_{+}}\left(e^{2 \lambda_{+}{ }^{t}}-1\right)
\end{array}
$$

hence 3.10 .

For any $\xi>0$ small enough, we define the sets

$$
\mathcal{K}_{k}^{\xi}:=\left\{(x, p): p-\lambda_{+}(x-2 k \pi)=\eta_{\epsilon},\left|p-\lambda_{-}(x-2 k \pi)\right| \leq \sigma_{\epsilon} \epsilon^{-\xi}\right\} .
$$

and

$$
\begin{aligned}
& \mathcal{H}_{k}^{\xi}:=\left\{(x, p): p=\eta_{\epsilon}+\lambda_{-}(x-2(k-1) \pi),\left|p-\lambda_{+}(x-2(k-1) \pi)\right| \leq \bar{\sigma}_{\epsilon} \epsilon^{-\xi}\right\}, k \geq 1 \\
& \mathcal{H}_{0}:=\left\{\left(-\pi, \wp_{0}^{*}(-\pi)\right)\right\}
\end{aligned}
$$

In the following propositions we provide some estimates on expected value and variance of $\hat{z}_{k}\left(S_{k}\right)$. 
Proposition 3.6. There exists $C>0$ such that, for any $\zeta>0, \xi, \epsilon$ small enough,

$$
\mathbf{1}_{\left(x_{k}, p_{k}\right) \in \mathcal{H}_{k}^{\xi}} \mathbf{P}_{T_{k}, x_{k}, p_{k}}\left\{\sigma_{r}\left(T_{k}, S_{k}\right)=\Theta\left(\sigma_{\epsilon}\right)\right\} \geq 1-e^{-C \epsilon^{-2 \zeta}}
$$

Proposition 3.7. Suppose that $\left(x_{k}, p_{k}\right) \in \mathcal{H}_{k}^{\xi}$ with $\xi$ small enough,

$$
\frac{\left|P_{k}\left(S_{k}\right)-\wp_{k}^{*}\left(X_{k}\left(S_{k}\right)\right)\right|}{\sigma_{r}\left(T_{k}, S_{k}\right)}=\mathcal{O}\left(\epsilon^{\theta}\right)
$$

Morover there exists $C>0$ such that, for any $\zeta>0, \xi, \epsilon$ small enough,

$$
\mathbf{1}_{\left(x_{k}, p_{k}\right) \in \mathcal{H}_{k}^{\xi}} \mathbf{P}_{T_{k}, x_{k}, p_{k}}\left\{\left|P_{k}\left(S_{k}\right)-\wp_{k}^{*}\left(X_{k}\left(S_{k}\right)\right)\right|=\mathcal{O}\left(\tilde{\sigma}_{\epsilon}\right)\right\} \geq 1-e^{-C \epsilon^{-2 \zeta}}
$$

Propositions 3.7 and 3.6 are proved in Section 6

In the following two propositions we give some estimates on the errors due to the linearizations. In Proposition 5.15 we provide an estimate from below of $T_{k+1}-S_{k}$.

Proposition 3.8. There exists $C>0$ such that, for any $\zeta, \epsilon$ small enough,

$$
\mathbf{1}_{\left(\bar{x}_{k}, \bar{p}_{k}\right) \in \mathcal{K}_{k}^{\xi}} \mathbf{P}_{S_{k}, \bar{x}_{k}, \bar{p}_{k}}\left\{\sup _{S_{k} \leq t \leq T_{k+1}}\left|\mathcal{Z}_{k}(t)\right| e^{-\lambda_{+}\left(t-S_{k}\right)}=\mathcal{O}\left(\eta_{\epsilon}^{2}\right)\right\} \geq 1-e^{-C \epsilon^{-2 \zeta}}
$$

and

$$
\mathbf{1}_{\left(\bar{x}_{k}, \bar{p}_{k}\right) \in \mathcal{K}_{k}^{\xi}} \mathbf{P}_{S_{k}, \bar{x}_{k}, \bar{p}_{k}}\left\{\sup _{S_{k} \leq t \leq T_{k+1}}\left|\mathcal{V}_{k}(t)\right|=\mathcal{O}\left(\eta_{\epsilon}^{2}\right)\right\} \geq 1-e^{-C \epsilon^{-2 \zeta}}
$$

Proposition 3.9. There exists $C>0$ such that, for any $\xi>0, \zeta, \epsilon$ small enough,

$$
\mathbf{1}_{\left(\bar{x}_{k}, \bar{p}_{k}\right) \in \mathcal{K}_{k}^{\xi}} \mathbf{P}_{S_{k}, \bar{x}_{k}, \bar{p}_{k}}\left\{T_{k+1} \geq S_{k}+\frac{1}{\lambda_{+}} \ln \left(\frac{\eta_{\epsilon}}{\sigma_{\epsilon}} \epsilon^{\xi}\right)\right\} \geq 1-e^{-C \epsilon^{-2 \zeta}}
$$

We will prove Propositions $3.10,3.8$ and 3.9 in Section 6

Proposition 3.10. There exists $C>0$ such that, for any $\xi>0, \zeta, \epsilon$ small enough,

$$
\mathbf{1}_{\left(x_{k}, p_{k}\right) \in \mathcal{H}_{k}^{\xi}} \mathbf{P}_{T_{k}, x_{k}, p_{k}}\left\{\left|z_{k}\left(S_{k}\right)-\hat{z}_{k}\left(S_{k}\right)\right|=\mathcal{O}\left(\eta_{\epsilon}^{2} \vee \sigma_{\epsilon} \eta_{\epsilon}^{-2} \epsilon^{1-2 \zeta}\right)\right\} \geq 1-e^{-C \epsilon^{-2 \zeta}}
$$

and

$$
\mathbf{1}_{\left(\bar{x}_{k-1}, \bar{p}_{k-1}\right) \in \mathcal{K}_{k-1}^{\xi}} \mathbf{P}_{S_{k-1}, \bar{x}_{k-1}, \bar{p}_{k-1}}\left\{\left|p\left(T_{k}\right)-\wp_{k}^{*}\left(x\left(T_{k}\right)\right)-\bar{v}_{k-1}\left(T_{k}\right)\right|=\mathcal{O}\left(\eta_{\epsilon}^{2}\right)\right\} \geq 1-e^{-C \epsilon^{-2 \xi}}
$$


We will denote by $\Phi(x)$ the function defined by

$$
\Phi(x):=\frac{1}{\sqrt{2 \pi}} \int_{x}^{+\infty} e^{-\frac{u^{2}}{2}} d u
$$

Lemma 3.11. There exists $C>0$ such that, for any $\xi, \epsilon$ small enough,

$$
\mathbf{1}_{\left(x_{k}, p_{k}\right) \in \mathcal{H}_{k}^{\xi}} \mathbf{P}_{T_{k}, x_{k}, p_{k}}\left\{\left|\hat{z}_{k}\left(S_{k}\right)\right| \leq \sigma_{\epsilon} \epsilon^{-\xi}\right\} \geq 1-e^{-C \epsilon^{-2 \xi}}
$$

Proof. Let us suppose $\left(x_{k}, p_{k}\right) \in \mathcal{H}_{k}^{\xi}$ and consider the event

$$
\mathbf{A}_{k}:=\left\{\sigma_{r}\left(T_{k}, S_{k}\right)=\Theta\left(\sigma_{\epsilon}\right),\left|P_{k}\left(S_{k}\right)-\wp_{k}^{*}\left(X_{k}\left(S_{k}\right)\right)\right|=\mathcal{O}\left(\tilde{\sigma}_{\epsilon}\right)\right\}
$$

for suitable $c, c^{\prime}, c^{\prime \prime}>0$, then

$$
\begin{array}{r}
\mathbf{P}_{T_{k}, x_{k}, p_{k}}\left\{\left|\hat{z}_{k}\left(S_{k}\right)\right| \geq \sigma_{\epsilon} \epsilon^{-\xi}\right\}=\mathbf{E}_{T_{k}, x_{k}, p_{k}}\left[\mathbf{P}\left\{\left|\hat{z}_{k}\left(S_{k}\right)\right| \geq \sigma_{\epsilon} \epsilon^{-\xi} \mid S_{k}\right\}\right] \\
\leq \mathbf{E}_{T_{k}, x_{k}, p_{k}}\left[\mathbf{1}_{\mathbf{A}_{k}} \mathbf{P}\left\{\left|\hat{z}_{k}\left(S_{k}\right)\right| \geq \sigma_{\epsilon} \epsilon^{-\xi} \mid S_{k}\right\}\right]+\mathbf{P}_{T_{k}, x_{k}, p_{k}}\left\{\mathbf{A}_{k}^{c}\right\}
\end{array}
$$

By Propositions 3.7 and 3.6

$$
\mathbf{P}_{T_{k}, x_{k}, p_{k}}\left\{\mathbf{A}_{k}^{c}\right\} \leq e^{-C \epsilon^{-2 \xi}}
$$

for a suitable $C>0, \xi$ small enough, and, by Lemma 3.5 , for any given $T_{k}$,

$$
\begin{aligned}
\mathbf{P}\left\{\left|\hat{z}_{k}\left(S_{k}\right)\right| \geq \sigma_{\epsilon} \epsilon^{-\xi} \mid S_{k}\right\}= & \Phi\left(\frac{\sigma_{\epsilon} \epsilon^{-\xi}-\left[P_{k}\left(S_{k}\right)-\wp_{k}^{*}\left(X_{k}\left(S_{k}\right)\right)\right]}{\sigma_{r}\left(T_{k}, S_{k}\right)}\right) \\
& +\Phi\left(\frac{\sigma_{\epsilon} \epsilon^{-\xi}-\left[P_{k}\left(S_{k}\right)+\wp_{k}^{*}\left(X_{k}\left(S_{k}\right)\right)\right]}{\sigma_{r}\left(T_{k}, S_{k}\right)}\right)
\end{aligned}
$$

where

$$
\mathbf{1}_{\mathbf{A}_{k}} \frac{\sigma_{\epsilon} \epsilon^{-\xi} \pm\left[P_{k}\left(S_{k}\right)-\wp_{k}^{*}\left(X_{k}\left(S_{k}\right)\right)\right]}{\sigma_{r}\left(T_{k}, S_{k}\right)} \geq c \epsilon^{-\xi}-c^{\prime} \frac{\tilde{\sigma}_{\epsilon}}{\sigma_{\epsilon}} \geq c^{\prime \prime} \epsilon^{-\xi}
$$

for suitable $c, c^{\prime}, c^{\prime \prime}>0$, since $\tilde{\sigma}_{\epsilon} / \sigma_{\epsilon}=\epsilon^{\theta}=o(1)$ as $\epsilon \rightarrow 0$. We have, thus,

$$
\mathbf{1}_{\mathbf{A}_{k}} \mathbf{P}\left\{\left|\hat{z}_{k}\left(S_{k}\right)\right| \geq \sigma_{\epsilon} \epsilon^{-\xi} \mid S_{k}\right\} \leq 2 \Phi\left(c^{\prime \prime} \epsilon^{-\xi}\right) \leq e^{-C \epsilon^{-2 \xi}}
$$

for some $C>0$, then the result follows from (3.23), (3.24) and (3.25).

Proposition 3.12. There exists $C>0$ such that, for any for any $\xi, \epsilon$ small enough,

$$
\mathbf{1}_{\left(x_{k}, p_{k}\right) \in \mathcal{H}_{k}^{\xi}} \mathbf{P}_{T_{k}, x_{k}, p_{k}}\left\{\left(x\left(S_{k}\right), p\left(S_{k}\right)\right) \in \mathcal{K}_{k}^{\xi}\right\} \geq 1-e^{-C \epsilon^{-2 \xi}}
$$

Proof. (3.26) follows directly from Lemma 3.11 and 3.19$)$, since, by $2.22, \epsilon=o\left(\sigma_{\epsilon}\right)$. 
Proposition 3.13. There exists $C>0$ such that, for any $\epsilon, \xi>0$ small enough,

$$
\mathbf{1}_{\left(x_{k}, p_{k}\right) \in \mathcal{H}_{k}^{\xi}} \mathbf{P}_{T_{k}, x_{k}, p_{k}}\left\{\sup _{S_{k} \leq t \leq T_{k+1}}\left|\mathcal{E}_{k}(t)\right| e^{-\lambda_{+}\left(t-S_{k}\right)}=\mathcal{O}\left(\eta_{\epsilon}^{2} \vee \sigma_{\epsilon} \eta_{\epsilon}^{-2} \epsilon^{1-2 \xi}\right)\right\} \geq 1-e^{-C \epsilon^{-2 \xi}}
$$

Proof. Let us suppose $\left(x_{k}, p_{k}\right) \in \mathcal{H}_{k}^{\xi}$ and define $\mathbf{B}_{k}:=\left\{\sup _{S_{k} \leq t \leq T_{k+1}}\left|\mathcal{Z}_{k}(t)\right| e^{-\lambda_{+}\left(t-S_{k}\right)}=\mathcal{O}\left(\eta_{\epsilon}^{2}\right)\right\}$, we have

$$
\begin{aligned}
\left|\mathbf{P}_{T_{k}, x_{k}, p_{k}}\left\{\mathbf{B}_{k}^{c}\right\}-\mathbf{P}\left\{\mathbf{B}_{k}^{c} \mid\left(x\left(S_{k}\right), p\left(S_{k}\right)\right) \in \mathcal{K}_{k}^{\xi}\right\}\right| \\
\leq\left|\mathbf{P}_{T_{k}, x_{k}, p_{k}}\left\{\mathbf{B}_{k}^{c},\left(x\left(S_{k}\right), p\left(S_{k}\right)\right) \in \mathcal{K}_{k}^{\xi}\right\}-\mathbf{P}\left\{\mathbf{B}_{k}^{c} \mid\left(x\left(S_{k}\right), p\left(S_{k}\right)\right) \in \mathcal{K}_{k}^{\xi}\right\}\right| \\
+\mathbf{P}_{T_{k}, x_{k}, p_{k}}\left\{\left(x\left(S_{k}\right), p\left(S_{k}\right)\right) \notin \mathcal{K}_{k}^{\xi}\right\} \\
\leq \mathbf{P}\left\{\mathbf{B}_{k}^{c} \mid\left(x\left(S_{k}\right), p\left(S_{k}\right)\right) \in \mathcal{K}_{k}^{\xi}\right\}\left|1-\mathbf{P}_{T_{k}, x_{k}, p_{k}}\left\{\left(x\left(S_{k}\right), p\left(S_{k}\right)\right) \in \mathcal{K}_{k}^{\xi}\right\}\right| \\
+\mathbf{P}_{T_{k}, x_{k}, p_{k}}\left\{\left(x\left(S_{k}\right), p\left(S_{k}\right)\right) \notin \mathcal{K}_{k}^{\xi}\right\} \\
\leq 2 \mathbf{P}_{T_{k}, x_{k}, p_{k}}\left\{\left(x\left(S_{k}\right), p\left(S_{k}\right)\right) \notin \mathcal{K}_{k}^{\xi}\right\}
\end{aligned}
$$

On the other hand,

$$
\mathbf{P}\left\{\mathbf{B}_{k}^{c} \mid\left(x\left(S_{k}\right), p\left(S_{k}\right)\right) \in \mathcal{K}_{k}^{\xi}\right\} \leq \sup _{\left(\bar{x}_{k}, \bar{p}_{k}\right) \in \mathcal{K}_{k}^{\xi}} \mathbf{P}_{S_{k}, \bar{x}_{k}, \bar{p}_{k}}\left\{\mathbf{B}_{k}^{c}\right\}
$$

then, from (3.16) and (3.26) it follows that there exists $C>0$ such that, for $\xi, \epsilon$ small enough,

$$
\mathbf{P}_{T_{k}, x_{k}, p_{k}}\left\{\mathbf{B}_{k}^{c}\right\} \leq e^{-C \epsilon^{-2 \xi}}
$$

thus, recalling that $\mathcal{E}_{k}(t) e^{-\lambda_{+}\left(t-S_{k}\right)}=\mathcal{Z}_{k}(t) e^{-\lambda_{+}\left(t-S_{k}\right)}+\left(z_{k}\left(S_{k}\right)-\hat{z}_{k}\left(S_{k}\right)\right)$, 3.27) follows directly from $(3.19)$ and $(3.29)$.

Proposition 3.14. There exists $c>0$ such that, for any $\epsilon>0$ small enough,

$$
\left|\mathbf{1}_{\left(x_{k}, p_{k}\right) \in \mathcal{H}_{k}^{\xi}} \mathbf{P}_{T_{k}, x_{k}, p_{k}}\left\{z_{k}\left(T_{k+1}\right) \gtrless 0\right\}-\frac{1}{2}\right| \leq c \epsilon^{\theta} .
$$

Proof. We assume $\left(x_{k}, p_{k}\right) \in \mathcal{H}_{k}^{\xi}$. We define the event

$$
\mathbf{C}_{k}:=\left\{\sup _{S_{k} \leq t \leq T_{k+1}}\left|\mathcal{E}_{k}(t)\right| e^{-\lambda_{+}\left(t-S_{k}\right)}=\mathcal{O}\left(v_{\epsilon}^{\xi}\right)\right\} .
$$

with $v_{\epsilon}^{\xi}:=\eta_{\epsilon}^{2} \vee \sigma_{\epsilon} \eta_{\epsilon}^{-2} \epsilon^{1-2 \xi}$. By 3.5 we have

$$
\begin{array}{r}
\mathbf{P}_{T_{k}, x_{k}, p_{k}}\left\{\mathbf{C}_{k}, z_{k}\left(T_{k+1}\right)>0\right\}=\mathbf{P}_{T_{k}, x_{k}, p_{k}}\left\{\mathbf{C}_{k}, \hat{z}_{k}\left(T_{k+1}\right)>-\mathcal{E}_{k}\left(T_{k+1}\right)\right\} \\
\leq \mathbf{P}_{T_{k}, x_{k}, p_{k}}\left\{\hat{z}_{k}\left(T_{k+1}\right)>-c v_{\epsilon} e^{\lambda_{+}\left(T_{k+1}-S_{k}\right)}\right\}
\end{array}
$$


For $\mathbf{A}_{k}$ as in the Proof of Lemma 3.11, we have

$$
\begin{aligned}
\mathbf{P}_{T_{k}, x_{k}, p_{k}} & \left\{\hat{z}_{k}\left(T_{k+1}\right)>-c v_{\epsilon} e^{\lambda_{+}\left(T_{k+1}-S_{k}\right)}\right\} \\
\leq & \mathbf{E}_{T_{k}, x_{k}, p_{k}}\left[\mathbf{1}_{\mathbf{A}_{k}} \mathbf{P}\left\{\hat{z}_{k}\left(T_{k+1}\right)>-c v_{\epsilon} e^{\lambda_{+}\left(T_{k+1}-S_{k}\right)} \mid S_{k}\right\}\right] \\
+ & +\mathbf{P}_{T_{k}, x_{k}, p_{k}}\left\{\mathbf{A}_{k}^{c}\right\} .
\end{aligned}
$$

By Lemma 3.5, for any given $T_{k}$ we have

$$
\begin{aligned}
& \mathbf{P}\left\{\hat{z}_{k}\left(T_{k+1}\right)>-c v_{\epsilon} e^{\lambda_{+}\left(T_{k+1}-S_{k}\right)} \mid S_{k}\right\} \\
& =\Phi\left(\frac{-\mu_{z, k}\left(T_{k+1}-S_{k}\right)-c v_{\epsilon} e^{\lambda_{+}\left(T_{k+1}-S_{k}\right)}}{\sigma_{z, k}\left(T_{k+1}-S_{k}\right)}\right)
\end{aligned}
$$

where, from 3.10 and 3.9 ,

$$
\begin{array}{r}
\mathbf{1}_{\mathbf{A}_{k}} \frac{\mu_{z, k}\left(T_{k+1}-S_{k}\right)+c v_{\epsilon} e^{\lambda_{+}\left(T_{k+1}-S_{k}\right)}}{\sigma_{z, k}\left(T_{k+1}-S_{k}\right)} \\
\leq \mathbf{1}_{\mathbf{A}_{k}} \frac{\left[P_{k}\left(S_{k}\right)-\wp_{k}^{*}\left(X_{k}\left(S_{k}\right)\right)\right]+c v_{\epsilon}}{\sigma_{r}\left(T_{k}, S_{k}\right)} \\
\leq c^{\prime} \frac{\tilde{\sigma}_{\epsilon}+v_{\epsilon}}{\sigma_{\epsilon}} \leq c^{\prime \prime} \epsilon^{\theta}
\end{array}
$$

for suitable $c^{\prime}, c^{\prime \prime}>0$, since, from (1.18), $v_{\epsilon}=o\left(\tilde{\sigma}_{\epsilon}\right)$. By 3.31, 3.32, 3.33) and (3.24) it follows that

$$
\mathbf{P}_{T_{k}, x_{k}, p_{k}}\left\{\mathbf{C}_{k}, z_{k}\left(T_{k+1}\right)>0\right\} \leq \Phi\left(-c^{\prime \prime} \epsilon^{\theta}\right)+e^{-C \epsilon^{-2 \xi}} \leq \frac{1}{2}+c^{\prime \prime \prime} \epsilon^{\theta}
$$

for suitable $C, c^{\prime \prime \prime}>0$, hence, by Proposition 3.13 , there exist $c, c^{\prime}, C>0$ such that

$$
\begin{array}{r}
\mathbf{P}_{T_{k}, x_{k}, p_{k}}\left\{z_{k}\left(T_{k+1}\right)>0\right\} \leq \mathbf{P}_{T_{k}, x_{k}, p_{k}}\left\{\mathbf{C}_{k}, z_{k}\left(T_{k+1}\right)>0\right\}+\mathbf{P}_{T_{k}, x_{k}, p_{k}}\left\{\mathbf{C}_{k}^{c}\right\} \\
\leq \frac{1}{2}+c\left(\epsilon^{\theta}+e^{-C \epsilon^{-2 \xi}}\right) \leq \frac{1}{2}+c^{\prime} \epsilon^{\theta}
\end{array}
$$

Analogously,

$$
\begin{array}{r}
\mathbf{P}_{T_{k}, x_{k}, p_{k}}\left\{\mathbf{C}_{k}, z_{k}\left(T_{k+1}\right)<0\right\} \\
=\mathbf{P}_{T_{k}, x_{k}, p_{k}}\left\{\mathbf{C}_{k}, \hat{z}_{k}\left(T_{k+1}\right)<-\mathcal{E}_{k}\left(T_{k+1}\right)\right\} \\
\leq \mathbf{P}_{T_{k}, x_{k}, p_{k}}\left\{\hat{z}_{k}\left(T_{k+1}\right)<c v_{\epsilon} e^{\lambda_{+}}\left(T_{k+1}-S_{k}\right)\right\} \\
\leq \frac{1}{2}+e^{-C \epsilon^{-2 \xi}}
\end{array}
$$

for some $C>0$, hence

$$
\begin{aligned}
\mathbf{P}_{T_{k}, x_{k}, p_{k}}\left\{z_{k}\left(T_{k+1}\right)<0\right\} \leq \mathbf{P}_{T_{k}, x_{k}, p_{k}}\left\{\mathbf{C}_{k}, z_{k}\left(T_{k+1}\right)<0\right\} & +\mathbf{P}_{T_{k}, x_{k}, p_{k}}\left\{\mathbf{C}_{k}^{c}\right\} \\
& \leq \frac{1}{2}+e^{-C^{\prime} \epsilon^{-2 \xi}}
\end{aligned}
$$

for some $C^{\prime}>0$, this concludes the proof of the Proposition.

Lemma 3.15. There exists $C>0$ such that, for any fixed $\epsilon, \xi>0$ small enough,

$$
\mathbf{1}_{\left(x_{k}, p_{k}\right) \in \mathcal{H}_{k}^{\xi}} \mathbf{P}_{T_{k}, x_{k}, p_{k}}\left\{T_{k+1} \leq S_{k}+\frac{1}{\lambda_{+}} \ln \left(\frac{\eta_{\epsilon}}{\sigma_{\epsilon}} \epsilon^{-\xi}\right)\right\} \geq 1-C \epsilon^{\xi}
$$


Proof. We assume $\left(x_{k}, p_{k}\right) \in \mathcal{H}_{k}^{\xi}$ for some $\xi$ small enough. Let $\mathbf{C}_{k}$ be as in 3.30 and define $\tau:=\ln \left(\eta_{\epsilon} \sigma_{\epsilon}^{-1} \epsilon^{-\xi}\right) / \lambda_{+}$, we have

$$
\begin{array}{r}
\mathbf{P}_{T_{k}, x_{k}, p_{k}}\left\{T_{k+1} \geq S_{k}+\tau\right\}=\mathbf{P}_{T_{k}, x_{k}, p_{k}}\left\{\sup _{0 \leq t \leq \tau}\left|z_{k}\left(S_{k}+t\right)\right| \leq \eta_{\epsilon}\right\} \\
\leq \mathbf{P}_{T_{k}, x_{k}, p_{k}}\left\{\sup _{0 \leq t \leq \tau}\left|z_{k}\left(S_{k}+t\right)\right| \leq \eta_{\epsilon}, \mathbf{C}_{k}\right\}+\mathbf{P}_{T_{k}, x_{k}, p_{k}}\left\{\mathbf{C}_{k}^{c}\right\}
\end{array}
$$

By (3.27) we know that there exists $C>0$ such that $\mathbf{P}_{T_{k}, x_{k}, p_{k}}\left\{\mathbf{C}_{k}^{c}\right\} \leq e^{-C \epsilon^{-2 \xi}}$ for any $\epsilon, \xi$ small enough. From the conditions in $(2.22)$ we have $\left(\eta_{\epsilon}^{2} \vee \sigma_{\epsilon} \eta_{\epsilon}^{-2} \epsilon^{1-2 \xi}\right) e^{\lambda_{+} \tau}=o\left(\eta_{\epsilon}\right)$, thus, by (3.5) and the definition of $\mathbf{C}_{k}$,

$$
\begin{aligned}
\mathbf{P}_{T_{k}, x_{k}, p_{k}}\left\{\sup _{0 \leq t \leq \tau}\left|z_{k}\left(S_{k}+t\right)\right| \leq \eta_{\epsilon}, \mathbf{C}_{k}\right\} \\
\leq \mathbf{P}_{T_{k}, x_{k}, p_{k}}\left\{\sup _{0 \leq t \leq \tau}\left|\hat{z}_{k}\left(S_{k}+t\right)\right| \leq 2 \eta_{\epsilon}\right\} \\
\quad=\mathbf{E}_{T_{k}, x_{k}, p_{k}}\left[\mathbf{P}\left\{\sup _{0 \leq t \leq \tau}\left|\hat{z}_{k}\left(S_{k}+t\right)\right| \leq 2 \eta_{\epsilon} \mid S_{k}\right\}\right]
\end{aligned}
$$

It is easy to show from Lemma 3.3. Proposition 3.7 and Proposition 3.6 that for any given $T_{k}$ there exists $C>0$ such that, for $\epsilon$ small enough,

$$
\mathbf{P}\left\{\sup _{0 \leq t \leq \tau}\left|\hat{z}_{k}\left(S_{k}+t\right)\right| \leq 2 \eta_{\epsilon} \mid S_{k}\right\} \leq C \epsilon^{\xi}
$$

then we get (3.34).

Corollary 3.16. If $\left(x_{k}, p_{k}\right) \in \mathcal{H}_{k}^{\xi}$ for some $\xi>0$ small enough then, for any fixed $\epsilon>0$ small enough, $k \geq 0, T_{k+1}-S_{k}$ is $\mathbf{P}_{T_{k}, x_{k}, p_{k}}$-a.s. finite.

Proof. It directly follows from the previous Lemma.

Lemma 3.17. There exists $C>0$ such that, for any $\epsilon, \xi>0$ small enough,

$$
\mathbf{1}_{\left(x_{k}, p_{k}\right) \in \mathcal{H}_{k}^{\xi}} \mathbf{P}_{T_{k}, x_{k}, p_{k}}\left\{\left|v_{k}\left(T_{k+1}\right)\right| \geq \bar{\sigma}_{\epsilon} \epsilon^{-\xi}\right\} \leq C \epsilon^{\xi}
$$

Proof. Assume that $\left(x_{k}, p_{k}\right) \in \mathcal{H}_{k}^{\xi}$, we first prove that there exists $C>0$ such that

$$
\mathbf{P}_{T_{k}, x_{k}, p_{k}}\left\{\left|\bar{v}_{k}\left(T_{k+1}\right)\right| \geq \bar{\sigma}_{\epsilon} \epsilon^{-\xi}\right\} \leq C \epsilon^{\xi}
$$

for any $\epsilon, \xi$ small enough.

Let us define the event $\mathbf{D}_{k}:=\left\{T_{k+1} \geq S_{k}+\frac{1}{\lambda_{+}} \ln \left(\eta_{\epsilon} \epsilon^{\xi} \sigma_{\epsilon}^{-1}\right)\right\}$, then

$$
\begin{aligned}
& \mathbf{P}_{T_{k}, x_{k}, p_{k}}\left\{\left|\bar{v}_{k}\left(T_{k+1}\right)-\mu_{v}\left(T_{k+1}-S_{k}\right)\right| \geq \epsilon^{1-\xi}\right\} \\
& \leq \mathbf{E}_{T_{k}, x_{k}, p_{k}}\left[\mathbf{1}_{\mathbf{D}_{k}} \mathbf{P}\left\{\left|\bar{v}_{k}\left(T_{k+1}\right)-\mu_{v}\left(T_{k+1}-S_{k}\right)\right| \geq \epsilon^{1-\xi} \mid S_{k}\right\}\right] \\
&+\mathbf{P}_{T_{k}, x_{k}, p_{k}}\left\{\mathbf{D}_{k}^{c}\right\}
\end{aligned}
$$


where, by Lemma 3.1 for any fixed $T_{k+1}$,

$$
\begin{array}{r}
\mathbf{P}\left\{\left|\bar{v}_{k}\left(T_{k+1}\right)-\mu_{v}\left(T_{k+1}-S_{k}\right)\right| \geq \epsilon^{1-\xi} \mid S_{k}\right\} \\
=2 \Phi\left(\frac{\epsilon^{1-\xi}}{\sigma_{v}\left(T_{k+1}-S_{k}\right)}\right)
\end{array}
$$

By (3.1) we have

$$
\mathbf{1}_{\mathbf{D}_{k}} \Phi\left(\frac{\epsilon^{1-\xi}}{\sigma_{v}\left(T_{k+1}-S_{k}\right)}\right) \leq \Phi\left(c \epsilon^{-\xi}\right) \leq c^{\prime} \epsilon^{-\xi} e^{-c^{\prime \prime} \epsilon^{-2 \xi}}
$$

for some $c, c^{\prime}, c^{\prime \prime}>0$, moreover, by Lemma 3.15 there exists $C$ such that

$$
\mathbf{P}_{T_{k}, x_{k}, p_{k}}\left\{\mathbf{D}_{k}^{c}\right\} \leq C \epsilon^{\xi}
$$

hence there exists $C^{\prime}>0$ such that

$$
\mathbf{P}_{T_{k}, \bar{x}, p_{k}}\left\{\left|\bar{v}_{k}\left(T_{k+1}\right)-\mu_{v}\left(T_{k+1}-S_{k}\right)\right| \geq \epsilon^{1-\xi}\right\} \leq C^{\prime} \epsilon^{\xi}
$$

By (3.1), $\mu_{v}\left(T_{k+1}-S_{k}\right)=\eta_{\epsilon} e^{\lambda_{-}\left(T_{k+1}-S_{k}\right)}$, thus, from 3.37) and the definition of $\bar{\sigma}_{\epsilon}$

$$
\mathbf{P}_{T_{k}, \bar{x}, p_{k}}\left\{\mu_{v}\left(T_{k+1}-S_{k}\right) \geq \bar{\sigma}_{\epsilon} \epsilon^{-\xi}\right\} \leq C \epsilon^{\xi}
$$

then (3.35) descends from (3.38) and (3.39) since $\epsilon=o\left(\bar{\sigma}_{\epsilon}\right)$. We recall now that $v_{k}(t)=\bar{v}_{k}(t)+\mathcal{V}_{k}(t)$, then (3.38) follows from (3.35) and (3.17) since $\eta_{\epsilon}^{2}=o\left(\bar{\sigma}_{\epsilon}\right)$.

We decompose the set $\mathcal{H}_{k}^{\xi}$ (see the definition in 3.12 ) in the two subests:

$$
\mathcal{H}_{k}^{\xi}=\mathcal{L}_{k}^{\xi} \cap \mathcal{M}_{k} \quad \text { for } \quad k \geq 1
$$

with

$$
\mathcal{L}_{k}^{\xi}:=\left\{(x, p):\left|p-\lambda_{+}(x-2(k-1) \pi)\right| \leq \bar{\sigma}_{\epsilon} \epsilon^{-\xi}\right\}
$$

and

$$
\mathcal{M}_{k}:=\left\{(x, p): p=\eta_{\epsilon}+\lambda_{-}(x-2(k-1) \pi)\right\}
$$

and recall that $\mathcal{H}_{0}=\left\{\left(-\pi, \wp_{0}^{*}(-\pi)\right)\right\}$.

Lemma 3.18. Suppose that $(x, p) \in \mathcal{H}_{k-1}$, then there exist $c, C>0$ such that

$$
\mathbf{P}_{T_{k-1}, x, p}\left\{\left(x\left(T_{k}\right), p\left(T_{k}\right)\right) \notin \mathcal{L}_{k}^{\xi}\right\} \leq C \epsilon^{\xi}
$$

and

$$
\left|\mathbf{P}_{T_{k-1}, x, p}\left\{\left(x\left(T_{k}\right), p\left(T_{k}\right)\right) \in \mathcal{H}_{k}^{\xi}\right\}-\frac{1}{2}\right| \leq c \epsilon^{\theta}
$$

for any $\epsilon$ small enough. 
Proof. (3.43) follows from (3.35) since $\left(x\left(T_{k}\right), p\left(T_{k}\right)\right) \notin \mathcal{L}_{k}^{\xi}$ if and only if $\left|v_{k-1}\left(T_{k}\right)\right| \geq \bar{\sigma}_{\epsilon} \epsilon^{-\xi}$. On the other hand, from Propositon 3.14 it follows that

$$
\left|\mathbf{P}_{T_{k-1}, x, p}\left\{\left(x\left(T_{k}\right), p\left(T_{k}\right)\right) \in \mathcal{M}_{k}\right\}-\frac{1}{2}\right| \leq c \epsilon^{\theta}
$$

since $\left(x\left(T_{k}\right), p\left(T_{k}\right)\right) \in \mathcal{M}_{k}$ if and only if $z_{k-1}\left(T_{k}\right)>0$. We have

$$
\begin{aligned}
& \mathbf{P}_{T_{k-1}, x, p}\left\{\left(x\left(T_{k}\right), p\left(T_{k}\right)\right) \in \mathcal{H}_{k}^{\xi}\right\} \\
& =\mathbf{P}_{T_{k-1}, x, p}\left\{\left(x\left(T_{k}\right), p\left(T_{k}\right)\right) \in \mathcal{M}_{k}\right\}-\mathbf{P}_{T_{k-1}, x, p}\left\{\left(x\left(T_{k}\right), p\left(T_{k}\right)\right) \in \mathcal{M}_{k} \cup\left(\mathcal{L}_{k}^{\xi}\right)^{c}\right\}
\end{aligned}
$$

then, from $(3.43)$

$$
\begin{aligned}
& \left|\mathbf{P}_{T_{k-1}, x, p}\left\{\left(x\left(T_{k}\right), p\left(T_{k}\right)\right) \in \mathcal{H}_{k}^{\xi}\right\}-\mathbf{P}_{T_{k-1}, x, p}\left\{\left(x\left(T_{k}\right), p\left(T_{k}\right)\right) \in \mathcal{M}_{k}\right\}\right| \\
& \leq \mathbf{P}_{T_{k-1}, x, p}\left\{\left(x\left(T_{k}\right), p\left(T_{k}\right)\right) \notin \mathcal{L}_{k}^{\xi}\right\} \leq C \epsilon^{\xi}
\end{aligned}
$$

then (3.44) follows from (3.45) and (3.46) since $\xi$ is arbitrary.

Lemma 3.19. Let $(x, p) \in \mathcal{H}_{0}$, then there exist $c, C>0$ such that

$$
\left(\frac{1}{2}-c \epsilon^{\theta}\right)^{k}-C \epsilon^{\xi} \leq \mathbf{P}_{T_{0}, x, p}\left\{\left(x\left(T_{k}\right), p\left(T_{k}\right)\right) \in \mathcal{H}_{k}^{\xi}\right\} \leq\left(\frac{1}{2}+c \epsilon^{\theta}\right)^{k}+C \epsilon^{\xi}
$$

for any $\epsilon$ small enough.

Proof. We prove the upper bound. For $(x, p) \in \mathcal{H}_{k-2}^{\xi}$ we have

$$
\begin{aligned}
& \mathbf{P}_{T_{k-2}, x, p}\left\{\left(x\left(T_{k}\right), p\left(T_{k}\right)\right) \in \mathcal{H}_{k}^{\xi}\right\} \\
& =\mathbf{E}_{T_{k-2}, x, p}\left[\mathbf{1}_{(x, p) \in \mathcal{M}_{k-1}} \mathbf{P}_{T_{k-1}, x, p}\left\{\left(x\left(T_{k}\right), p\left(T_{k}\right)\right) \in \mathcal{H}_{k}^{\xi}\right\}\right] \\
& \leq \mathbf{E}_{T_{k-2}, x, p}\left[\mathbf{1}_{(x, p) \in \mathcal{H}_{k-1}^{\xi}} \mathbf{P}_{T_{k-1}, x, p}\left\{\left(x\left(T_{k}\right), p\left(T_{k}\right)\right) \in \mathcal{H}_{k}^{\xi}\right\}\right]+\mathbf{P}_{T_{k-2}, x, p}\left\{\left(x\left(T_{k-1}\right), p\left(T_{k-1}\right)\right) \notin \mathcal{L}_{k-1}\right\} \\
& \leq\left(\frac{1}{2}+c \epsilon^{\theta}\right) \mathbf{P}_{T_{k-2}, x, p}\left\{\left(x\left(T_{k-1}\right), p\left(T_{k-1}\right)\right) \in \mathcal{H}_{k-1}^{\xi}\right\}+C \epsilon^{\xi} \\
& \leq\left(\frac{1}{2}+c \epsilon^{\theta}\right)^{2}+C \epsilon^{\xi}
\end{aligned}
$$

where the two last inequalities follow from (3.43) and (3.44). By repeating $k$ times this argument we find that, if $(x, p) \in \mathcal{H}_{0}$,

$$
\mathbf{P}_{T_{0}, x, p}\left\{\left(x\left(T_{k}\right), p\left(T_{k}\right)\right) \in \mathcal{H}_{k}^{\xi}\right\} \leq\left(\frac{1}{2}+c \epsilon^{\theta}\right)^{k}+C \epsilon^{\xi} \sum_{i=0}^{k-2}\left(\frac{1}{2}+c \epsilon^{\theta}\right)^{i} \leq\left(\frac{1}{2}+c \epsilon^{\theta}\right)^{k}+C^{\prime} \epsilon^{\xi}
$$

The lower bound follows from the same argument.

Lemma 3.20. Let $(x, p) \in \mathcal{H}_{0}$, then there exists $C>0$ such that

$$
\mathbf{P}_{T_{0}, x, p}\left\{\left(x\left(T_{k}\right), p\left(T_{k}\right)\right) \notin \mathcal{L}_{k}^{\xi}\right\} \leq C \epsilon^{\xi}
$$

for any $\epsilon$ small enough. 
Proof. For $(x, p) \in \mathcal{H}_{k-2}^{\xi}$ we have

$$
\begin{gathered}
\mathbf{P}_{T_{k-2}, x, p}\left\{\left(x\left(T_{k}\right), p\left(T_{k}\right)\right) \notin \mathcal{L}_{k}^{\xi}\right\}= \\
=\mathbf{E}_{T_{k-2}, x, p}\left[\mathbf{1}_{(x, p) \in \mathcal{M}_{k-1}} \mathbf{P}_{T_{k-1}, x, p}\left\{\left(x\left(T_{k}\right), p\left(T_{k}\right)\right) \notin \mathcal{L}_{k}^{\xi}\right\}\right] \\
\leq \mathbf{E}_{T_{k-2}, x, p}\left[\mathbf{1}_{(x, p) \in \mathcal{H}_{k-1}^{\xi}} \mathbf{P}_{T_{k-1}, x, p}\left\{\left(x\left(T_{k}\right), p\left(T_{k}\right)\right) \notin \mathcal{L}_{k}\right\}\right]+\mathbf{P}_{T_{k-2}, x, p}\left\{\left(x\left(T_{k-1}\right), p\left(T_{k-1}\right)\right) \notin \mathcal{L}_{k-1}\right\} \\
\leq C \epsilon^{\xi} \mathbf{P}_{T_{k-2}, x, p}\left\{\left(x\left(T_{k-1}\right), p\left(T_{k-1}\right)\right) \in \mathcal{H}_{k-1}^{\xi}\right\}+C \epsilon^{\xi} \\
\leq C \epsilon^{\xi}\left[\left(\frac{1}{2}+c \epsilon^{\theta}\right)+1\right]
\end{gathered}
$$

the last two inequalities descending from (3.43) and (3.44). By repeating $k$ times this argument we find that, for $(x, p) \in \mathcal{H}_{0}$,

$$
\mathbf{P}_{T_{0}, x, p}\left\{\left(x\left(T_{k}\right), p\left(T_{k}\right)\right) \notin \mathcal{L}_{k}^{\xi}\right\} \leq C \epsilon^{\xi} \sum_{i=0}^{k-1}\left(\frac{1}{2}+c \epsilon^{\theta}\right)^{i} \leq C^{\prime} \epsilon^{\xi}
$$

this concludes the proof of the Lemma.

Conclusion of the Proof of Theorem 1.1. The processes $z_{k}(t)$ are well defined only for $k \leq \mathcal{N}$, then we set

$$
z_{k}(t) \equiv 0 \quad \text { for } \quad k \geq \mathcal{N}+1
$$

Let $(x, p) \in \mathcal{H}_{0}$, then we prove that there exists $C>0$ such that

$$
\left|\mathbf{P}_{T_{0}, x, p}\{\mathcal{N}=k\}-\left(\frac{1}{2}+c \epsilon^{\theta}\right)^{k+1}\right| \leq C \epsilon^{\xi}
$$

for any $\epsilon$ small enough. We have

$$
\begin{aligned}
& \mathbf{P}_{T_{0}, x, p}\{\mathcal{N}=k\}=\mathbf{P}_{T_{0}, x, p}\left\{z_{k}\left(T_{k+1}\right)<0, z_{k-1}\left(T_{k}\right)>0, \ldots, z_{0}\left(T_{1}\right)>0\right\} \\
& =\mathbf{P}_{T_{0}, x, p}\left\{z_{k}\left(T_{k+1}\right)<0, z_{k-1}\left(T_{k}\right)>0\right\} \\
& =\mathbf{P}_{T_{0}, x, p}\left\{\left(x\left(T_{k+1}\right), p\left(T_{k+1}\right)\right) \notin \mathcal{M}_{k+1},\left(x\left(T_{k}\right), p\left(T_{k}\right)\right) \in \mathcal{M}_{k}\right\} \\
& =\mathbf{E}_{T_{0}, x, p}\left[\mathbf{1}_{(x, p) \in \mathcal{M}_{k}} \mathbf{P}_{T_{k}, x, p}\left\{\left(x\left(T_{k+1}\right), p\left(T_{k+1}\right)\right) \notin \mathcal{M}_{k+1}\right\}\right] \\
& \leq \mathbf{E}_{T_{0}, x, p}\left[\mathbf{1}_{(x, p) \in \mathcal{H}_{k}^{\xi}} \mathbf{P}_{T_{k}, x, p}\left\{\left(x\left(T_{k+1}\right), p\left(T_{k+1}\right)\right) \notin \mathcal{M}_{k+1}\right\}\right]+\mathbf{P}_{T_{0}, x, p}\left\{\left(x\left(T_{k}\right), p\left(T_{k}\right)\right) \notin \mathcal{L}_{k}^{\xi}\right\} \\
& \leq\left(\frac{1}{2}+c \epsilon^{\theta}\right) \mathbf{P}_{T_{0}, x, p}\left\{\left(x\left(T_{k}\right), p\left(T_{k}\right)\right) \in \mathcal{H}_{k}^{\xi}\right\}+C \epsilon^{\xi} \\
& \leq\left(\frac{1}{2}+c \epsilon^{\theta}\right)\left[\left(\frac{1}{2}+c \epsilon^{\theta}\right)^{k}+C \epsilon^{\xi}\right]+C \epsilon^{\xi} \\
& \leq\left(\frac{1}{2}+c \epsilon^{\theta}\right)^{k+1}+C^{\prime} \epsilon^{\xi}
\end{aligned}
$$

the last three inequalities descenging from (3.45), $(3.50)$ and (3.47). This yelds the upper bound in (3.53, the lower bound can be obtained by an analogous argument. Thus Theorem 1.1 follows from 3.53 . 
Proof of Proposition 1.3. For $\xi>0$ we define the set

$$
\mathcal{Q}_{k}^{\xi}:=\left\{(x, p): x \leq 2 k \pi-\frac{\eta_{\epsilon}}{\left(\lambda_{+}-\lambda_{-}\right)}\left(1-\frac{\bar{\sigma}_{\epsilon}}{\eta_{\epsilon}} \epsilon^{-\xi}\right), p<-\frac{\lambda_{+} \eta_{\epsilon}}{\left(\lambda_{+}-\lambda_{-}\right)}\left(1-\frac{\left|\lambda_{-}\right| \bar{\sigma}_{\epsilon} \epsilon^{-\xi}}{\lambda_{+} \eta_{\epsilon}}\right)\right\}
$$

We prove that, for $(x, p) \in \mathcal{H}_{0}$,

$$
\lim _{\epsilon \rightarrow 0} \mathbf{P}_{T_{0}, x, p}\left\{\left(x\left(T_{\mathcal{N}+1}\right), p\left(T_{\mathcal{N}+1}\right)\right) \notin \mathcal{Q}_{\mathcal{N}}^{\xi}\right\}=0
$$

We have

$$
\begin{aligned}
& \mathbf{P}_{T_{0}, x, p}\left\{\left(x\left(T_{\mathcal{N}+1}\right), p\left(T_{\mathcal{N}+1}\right)\right) \notin \mathcal{Q}_{\mathcal{N}}^{\xi}\right\} \\
& =\mathbf{P}_{T_{0}, x, p}\left\{\left(x\left(T_{\mathcal{N}+1}\right), p\left(T_{\mathcal{N}+1}\right)\right) \notin \mathcal{Q}_{\mathcal{N}}^{\xi}, z_{\mathcal{N}}\left(T_{\mathcal{N}+1}\right)<0, z_{\mathcal{N}-1}\left(T_{\mathcal{N}}\right)>0\right\} \\
& =\mathbf{E}_{T_{0}, x, p}\left[\mathbf{1}_{(x, p) \in \mathcal{M}_{\mathcal{N}}} \mathbf{P}_{T_{\mathcal{N}, x, p}}\left\{\left(x\left(T_{\mathcal{N}+1}\right), p\left(T_{\mathcal{N}+1}\right)\right) \notin \mathcal{Q}_{\mathcal{N}}^{\xi}, z_{\mathcal{N}}\left(T_{\mathcal{N}+1}\right)=-\eta_{\epsilon}\right\}\right]
\end{aligned}
$$

it is easy to check from the definition of $\mathcal{Q}_{k}^{\xi}$ that

$$
\left\{\left(x\left(T_{\mathcal{N}+1}\right), p\left(T_{\mathcal{N}+1}\right)\right) \notin \mathcal{Q}_{\mathcal{N}}^{\xi}, z_{\mathcal{N}}\left(T_{\mathcal{N}+1}\right)=-\eta_{\epsilon}\right\}=\left\{\left|v_{\mathcal{N}}\left(T_{\mathcal{N}+1}\right)\right| \geq \bar{\sigma}_{\epsilon} \epsilon^{-\xi}\right\}
$$

then

$$
\begin{aligned}
& \mathbf{P}_{T_{0}, x, p}\left\{\left(x\left(T_{\mathcal{N}+1}\right), p\left(T_{\mathcal{N}+1}\right)\right) \notin \mathcal{Q}_{\mathcal{N}}^{\xi}\right\}=\mathbf{E}_{T_{0}, x, p}\left[\mathbf{1}_{(x, p) \in \mathcal{M}_{\mathcal{N}}} \mathbf{P}_{T_{\mathcal{N}}, x, p}\left\{\left|v_{\mathcal{N}}\left(T_{\mathcal{N}+1}\right)\right| \geq \bar{\sigma}_{\epsilon} \epsilon^{-\xi}\right\}\right] \\
& \left.\leq \mathbf{E}_{T_{0}, x, p}\left[\mathbf{1}_{(x, p) \in \mathcal{H}_{\mathcal{N}}^{\xi}} \mathbf{P}_{T_{\mathcal{N}, x, p}}\left\{\left|v_{\mathcal{N}}\left(T_{\mathcal{N}+1}\right)\right| \geq \bar{\sigma}_{\epsilon} \epsilon^{-\xi}\right\}\right]+\mathbf{P}_{T_{0}, x, p}\left\{\left(x\left(T_{\mathcal{N}}\right), p\left(T_{\mathcal{N}}\right)\right)\right) \notin \mathcal{L}_{\mathcal{N}}^{\xi}\right\}
\end{aligned}
$$

then 3.54 follows from $3.35,3.50$ and 1.22 .

\section{Deterministic paths}

In this Section we study the qualitative behavior of the orbits of the system (2.5) lying in a neighborhood of the heteroclinic path. We recall that $\wp_{k}^{*}(x)$ is the heteroclinic orbit defined in $(2(k-1) \pi, 2 k \pi)$. We have the following result.

Lemma 4.1. Let us fix $\delta>0$ small enough, then, for any $2(k-1) \pi<x \leq 2(k-1) \pi+\delta$,

$$
\wp_{k}^{*}(x)=\lambda_{+}(1+\mathcal{O}(\delta))(x-2(k-1) \pi)
$$

whereas, for any $2 k \pi-\delta \leq x<2 k \pi$

$$
\wp_{k}^{*}(x)=\lambda_{-}(1+\mathcal{O}(\delta))(x-2 k \pi)
$$

Proof. It follows directly from 1.11.

We denote by $\wp_{k}(x)$ a generic orbit in the phase plane close enough to $\wp_{k}^{*}(x)$ in $(2(k-1) \pi, 2 k \pi)$ in the following sense. We fix $\eta>0$ small enough and define $x_{k}, x_{k}^{\prime} \in(2(k-1) \pi, 2 k \pi), x_{k}<x_{k}^{\prime}$ such that

$$
x_{k}-2(k-1) \pi=\Theta(\eta) \quad \text { and } \quad 2 k \pi-x_{k}^{\prime}=\Theta(\eta) \quad \text { as } \quad \eta \rightarrow 0
$$

we suppose

$$
\left|\wp_{k}\left(x_{k}\right)-\wp_{k}^{*}\left(x_{k}\right)\right|=o(\eta) \quad \text { as } \quad \eta \rightarrow 0
$$

then, as we will see in the following Lemma, $\wp_{k}(x)$ is well defined in $\left[x_{k}, x_{k}^{\prime}\right]$. 
Lemma 4.2. For $x_{k}, x_{k}^{\prime}$ as in 4.3) and $\wp_{k}(x)$ satisfying 4.4], we define $\rho_{k}(x):=\wp_{k}(x)-\wp_{k}^{*}(x)$. Let $f_{k}^{\delta}(x):=\delta^{-\frac{\beta}{\lambda^{2}}+} e^{\int_{\delta}^{x} \frac{V^{\prime}(u)}{\xi_{k}^{*}(u)} d u}$ then, for any $\delta>\eta$ small enough,

$$
\begin{array}{rlr}
\rho_{k}(x)=\rho_{k}\left(x_{k}\right)\left(\frac{x_{k}}{x}\right)^{\frac{\beta}{\lambda^{2}}}(1+\mathcal{O}(\delta)) & \text { for } & x_{k} \leq x \leq 2(k-1) \pi+\delta \\
\rho_{k}(x)=\rho_{k}\left(x_{k}\right) x_{k}^{\frac{\beta}{\lambda^{2}}} f_{k}^{\delta}(x)(1+\mathcal{O}(\delta)) & \text { for } & 2(k-1) \pi+\delta \leq x \leq 2 k \pi-\delta
\end{array}
$$

and

$$
\rho_{k}(x)=\rho_{k}\left(x_{k}\right) \frac{x_{k}^{\frac{\beta}{\lambda^{2}}}}{(2 k \pi-x)^{\frac{\beta}{\lambda^{2}}}} f_{k}^{\delta}(2 k \pi-\delta)(1+\mathcal{O}(\delta)) \quad \text { for } \quad 2 k \pi-\delta \leq x \leq x_{k}^{\prime}
$$

Proof. Because of the periodicity of the dynamics, it is sufficient to prove the result for $k=1$. In order to lighten the notation, we omit the index 1 in $\wp_{1}(x), \wp_{1}^{*}(x)$, etc.

We define $x_{*}:=\inf \left\{x \geq x_{1}:|\rho(x)| \geq \eta\right\}$, thus, by 1.5),

$$
\rho^{\prime}(x)=\frac{V^{\prime}(x)}{\wp^{*}(x) \wp(x)}=\frac{V^{\prime}(x)}{\wp^{*}(x)\left(\wp^{*}(x)+\rho(x)\right)}
$$

then

$$
\rho(x)=\rho\left(x_{1}\right) e^{\int_{x_{1}}^{x} \frac{V^{\prime}(u)}{b^{*}(u)\left(\varphi^{*}(u)+\rho(u)\right)} d u} \quad \text { for } \quad x \geq x_{1}
$$

hence there exists a function $g(\cdot)$ such that

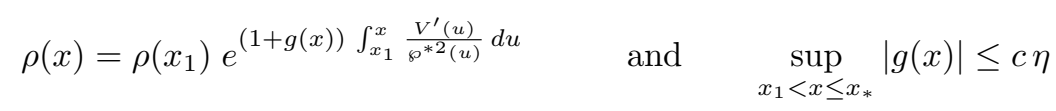

for some $c>0$. We fix $\delta>0$ small enough, $\delta>\eta$, then, by (1.9), for any $k \geq 0$,

$$
V^{\prime}(x)=-\beta(x-2 k \pi)(1+\mathcal{O}(\delta)) \quad \text { for } \quad|x-2 k \pi| \leq \delta
$$

By 4.1] and 4.11, there exists $\bar{g}(x)$, with $\sup _{0 \leq x \leq \delta}|\bar{g}(x)|=\mathcal{O}(\delta)$ as $\delta \rightarrow 0$, such that

$$
\int_{x_{1}}^{x} \frac{V^{\prime}(u)}{\wp^{* 2}(u)} d u=-\frac{\beta}{\lambda_{+}^{2}} \ln \left(\frac{x}{x_{1}}\right)+\bar{g}(x) \quad \text { for } \quad x_{1} \leq x \leq \delta
$$

and, by (4.2) and (4.11) there exists $\tilde{g}(x)$, with $\sup _{2 \pi-\delta \leq x \leq 2 \pi}|\tilde{g}(x)|=\mathcal{O}(\delta)$ as $\delta \rightarrow 0$, such that

$$
\int_{2 \pi-\delta}^{x} \frac{V^{\prime}(u)}{\wp^{* 2}(u)} d u=-\frac{\beta}{\lambda_{-}^{2}} \ln \left(\frac{2 \pi-x}{\delta}\right)+\tilde{g}(x) \quad \text { for } \quad 2 \pi-\delta \leq x \leq 2 \pi
$$

Since $x_{1}=o(\delta)$, from $4.9,44.13$ and 4.12 we gather

$$
\rho(x)=\rho\left(x_{1}\right) e^{\bar{g}(x)(1+g(x))}\left(\frac{x_{1}}{x}\right)^{\frac{\beta}{\lambda_{+}^{2}}(1+g(x))} \quad \text { for } \quad x_{1} \leq x \leq \delta,
$$

then, in particular, from 4.4,

$$
\sup _{x_{1} \leq x \leq \delta \wedge x_{*}}|\rho(x)| \leq\left|\rho\left(x_{1}\right)\right|=o(\eta)
$$


thus $x_{*}>\delta$ then 4.5 follows from 4.14. From 4.5) we have, thus

$$
\rho(x)=\rho\left(x_{1}\right) x_{1}^{\frac{\beta}{\lambda^{2}}} f^{\delta}(x)(1+\mathcal{O}(\delta)) \quad \text { for } \quad \delta \leq x \leq(2 \pi-\delta) \wedge x_{*}
$$

Since $f^{\delta}(x)$ does not depend on $\eta$, we have in particular that $\sup _{\delta \leq x \leq 2 \pi-\delta}|\rho(x)|=o(\eta)$, hence $x_{*}>2 \pi-\delta$, thus (4.6) follows. Finally

$$
\rho(x)=\rho(2 \pi-\delta) e^{\tilde{g}(x)(1+g(x))}(2 \pi-x)^{-\frac{\beta}{\lambda^{2}}(1+g(x))} \quad \text { for } \quad 2 \pi-\delta \leq x<2 \pi
$$

thus

$$
\begin{array}{r}
\sup _{2 \pi-\delta \leq x \leq x_{1}^{\prime} \wedge x_{*}}|\rho(x)|=\left|\rho\left(x_{1}^{\prime}\right)\right|=|\rho(2 \pi-\delta)| \mathcal{O}\left(\eta^{-\frac{\beta}{\lambda^{2}}}\right) \\
=\left|\rho\left(x_{1}\right)\right| \mathcal{O}\left(\eta^{\beta\left(\frac{1}{\lambda_{+}^{2}}-\frac{1}{\lambda^{2}}\right)}\right)=o(\eta) \quad \text { as } \quad \eta \rightarrow 0
\end{array}
$$

since $\frac{\beta}{\lambda_{+}^{2}}>\frac{\beta}{\lambda_{-}^{2}}$. We have, thus, $x_{*}>x_{1}^{\prime}$, then 4.7 follows from 4.16 and 4.17.

Remark 4.3. Notice that

$$
\rho_{k}(x)=o(\eta) \quad \text { for any } \quad x_{k} \leq x \leq x_{k}^{\prime}
$$

and, by 4.18,

$$
\rho\left(x_{k}^{\prime}\right)=\left|\rho\left(x_{k}\right)\right| \mathcal{O}\left(\eta^{\frac{2+\theta}{1+\theta} \theta}\right) \quad \text { as } \quad \eta \rightarrow 0
$$

since $\frac{\beta}{\lambda_{+}^{2}}-\frac{\beta}{\lambda_{-}^{2}}=\frac{\gamma}{\beta} \sqrt{\gamma^{2}+4 \beta}=\frac{2+\theta}{1+\theta} \theta$.

Lemma 4.4. For $x_{k}, x_{k}^{\prime}$ as in (4.3) and $\wp_{k}(x)$ satisfying 4.4,

$$
\sup _{x_{k} \leq x \leq x_{k}^{\prime}}\left|\frac{d}{d x} \wp_{k}(x)\right|=\mathcal{O}(1) \quad \text { as } \quad \eta \rightarrow 0
$$

Proof. $\wp_{k}(x)$ verifies the equation

$$
\frac{d}{d x} \wp_{k}(x)=-\frac{V^{\prime}(x)}{\wp_{k}(x)}-\gamma
$$

then it is sufficient to prove that $V^{\prime}(x) / \wp_{k}(x)$ is uniformly bounded in $\eta$ in a neighborhood of $x_{k}$ and $x_{k}^{\prime}$. Thus the result easily follows by expanding $V^{\prime}(x)$ and $\wp_{k}(x)$ in a neighborhood of $x_{k}$ and $x_{k}^{\prime}$ and by using Lemma 4.1 and Lemma 4.2 .

By similar arguments can be proved the following Lemma.

Lemma 4.5. For $x_{k}, x_{k}^{\prime}$ as in 4.3 and $\wp_{k}(x)$ satisfying 4.4 we have

$$
\left|\frac{d}{d x} \wp_{k}(x)-\frac{d}{d x} \wp_{k}^{*}(x)\right| \leq\left|\wp_{k}\left(x_{k}\right)-\wp_{k}^{*}\left(x_{k}\right)\right| \eta^{-1} \quad \text { for } \quad x_{k} \leq x \leq x_{k}^{\prime}
$$


We define, now, the functionals

$$
\Sigma_{r}^{(n)}[\wp, \bar{x}](x):=\int_{\bar{x}}^{x} \frac{d u}{\wp(u)} e^{n \int_{u}^{x} \frac{V^{\prime}\left(u^{\prime}\right)}{\wp^{2}\left(u^{\prime}\right)} d u^{\prime}}
$$

and

$$
\Sigma_{y}^{(n)}[\wp, \bar{x}](x):=\wp^{n}(x) \int_{\bar{x}}^{x} \frac{d u}{\wp(u)}\left[\int_{u}^{x} \frac{d u^{\prime}}{\wp^{2}\left(u^{\prime}\right)} e^{\int_{u}^{u^{\prime}} \frac{V^{\prime}\left(u^{\prime \prime}\right)}{\wp^{2}\left(u^{\prime \prime}\right)} d u^{\prime \prime}}\right]^{n}
$$

$n \in \mathbb{N}, x \geq \bar{x}$. In the rest of the Section we provide some estimates on $\Sigma_{r}^{(n)}[\wp, \bar{x}](\cdot)$ and $\Sigma_{y}^{(n)}[\wp, \bar{x}](\cdot)$ that are fundamental for the study of the variances in Section 5 .

For $\eta, x_{k}, x_{k}^{\prime}, \wp_{k}(x)$ verifying the conditions in (4.3) and (4.4), we prove the following Lemma.

Lemma 4.6. For any $k \geq 1, n \in \mathbb{N}$ we have

$$
\sup _{x_{k} \leq x \leq x_{k}^{\prime}} \frac{\Sigma_{r}^{(n)}\left[\wp_{k}, x_{k}\right](x)}{\rho_{k}(x)^{n}} \rho_{k}\left(x_{k}\right)^{n} x_{k}^{n(1+\theta)}=\mathcal{O}(1) \quad \text { as } \quad \eta \rightarrow 0
$$

moreover

$$
\Sigma_{r}^{(n)}\left[\wp_{k}, x_{k}\right]\left(x_{k}^{\prime}\right) \frac{\rho_{k}\left(x_{k}\right)^{n} x_{k}^{n(1+\theta)}}{\rho_{k}\left(x_{k}^{\prime}\right)^{n}}=\Theta(1) \quad \text { as } \quad \eta \rightarrow 0
$$

Proof. As before, we prove the statement for $k=1$ and omit the index 1 in the notation. At first we prove that

$$
\left|\frac{\Sigma_{r}^{(n)}\left[\wp, x_{1}\right](x)}{\rho(x)^{n} F_{n}\left[\wp, x_{1}\right](x)}-1\right|=o(\eta) \quad \text { with } \quad F_{n}\left[\wp, x_{1}\right](x):=\int_{x_{1}}^{x} \frac{d u}{\wp(u) \rho(u)^{n}}
$$

From 4.8 we have

$$
\rho^{\prime}(x)=\frac{V^{\prime}(x)}{\wp(x)(\wp(x)-\rho(x))}
$$

then

$$
\rho(x)=\rho(u) e^{\int_{u}^{x} \frac{V^{\prime}\left(u^{\prime}\right)}{\wp\left(u^{\prime}\right)\left(\wp\left(u^{\prime}\right)-\rho\left(u^{\prime}\right)\right)} d u^{\prime}} \quad \text { for any } \quad x_{1} \leq u \leq x
$$

thus, from 4.20 there exists $\hat{g}(x)$ such that

$$
\rho(x)=\rho(u) e^{(1+\hat{g}(x)) \int_{u}^{x} \frac{V^{\prime}\left(u^{\prime}\right)}{\wp^{2}\left(u^{\prime}\right)} d u^{\prime}} \quad \text { and } \quad \sup _{x_{1} \leq x \leq x_{1}^{\prime}}|\hat{g}(x)|=o(\eta)
$$

it follows that

$$
\sup _{x_{1} \leq u \leq x \leq x_{1}^{\prime}}\left|\frac{\rho(u)^{n}}{\rho(x)^{n}} e^{n \int_{u}^{x} \frac{V^{\prime}\left(u^{\prime}\right)}{\wp\left(u^{\prime}\right)^{2}} d u^{\prime}}-1\right|=o(\eta)
$$

this yields 4.28.

Let $\delta$ be small enough, $x \in\left[x_{1}, \delta\right]$, then by 4.1) and (4.5),

$$
\begin{array}{r}
F_{n}\left[\wp, x_{1}\right](x)=(1+\mathcal{O}(\delta)) \frac{x_{1}^{-\frac{n \beta}{\lambda_{+}^{2}}}}{\lambda_{+} \rho\left(x_{1}\right)^{n}} \int_{x_{1}}^{x} u^{-1+\frac{n \beta}{\lambda_{+}^{2}}} d u \\
=(1+\mathcal{O}(\delta)) \frac{\lambda_{+}}{n \beta \rho\left(x_{1}\right)^{n}}\left[\left(\frac{x}{x_{1}}\right)^{\frac{n \beta}{\lambda_{+}^{2}}}-1\right] \quad \text { for } \quad x_{1} \leq x \leq \delta
\end{array}
$$


Let, now, $x \in[\delta, 2 \pi-\delta]$, then $F_{n}\left[\wp, x_{1}\right](\delta)=F_{n}\left[\wp, x_{1}\right](\delta)+F_{n}[\wp, \delta](x)$ with, by 4.6 and 4.20),

$$
F_{n}[\wp, \delta](x)=(1+\mathcal{O}(\delta)) \frac{x_{1}^{-\frac{n \beta}{\lambda_{+}^{2}}}}{\rho\left(x_{1}\right)^{n}} \int_{\delta}^{x} \frac{d u}{\wp^{*}(u) f^{\delta}(u)^{n}} \quad \text { for } \quad \delta \leq x \leq 2 \pi-\delta
$$

with $f^{\delta}(x)$ as in Lemma 4.2 Finally, for $x \in\left[2 \pi-\delta, x_{1}^{\prime}\right], F_{n}\left[\wp, x_{1}\right](x)=F_{n}\left[\wp, x_{1}\right](2 \pi-\delta)+F_{n}[\wp, 2 \pi-$ $\delta](x)$, with, by 4.2 and 4.7 ,

$$
\begin{gathered}
F_{n}[\wp, 2 \pi-\delta](x)=(1+\mathcal{O}(\delta)) \frac{x_{1} \frac{n \beta}{\lambda^{2}}}{\left|\lambda_{-}\right| f^{\delta}(2 \pi-\delta)^{n} \rho\left(x_{1}\right)^{n}} \int_{2 \pi-x}^{\delta} u^{-1+\frac{n \beta}{\lambda^{2}}} d u \\
=(1+\mathcal{O}(\delta)) \frac{\left|\lambda_{-}\right| x_{1}{ }^{-\frac{n \beta}{\lambda_{+}^{2}}}}{n \beta f^{\delta}(2 \pi-\delta)^{n} \rho\left(x_{1}\right)^{n}}\left[\delta^{\frac{n \beta}{\lambda^{2}}}-(2 \pi-x)^{\frac{n \beta}{\lambda^{2}}}\right] \quad \text { for } \quad 2 \pi-\delta \leq x \leq x_{1}^{\prime}
\end{gathered}
$$

From 4.34, 4.35 and 4.37) we have that, for any $x \in\left[x_{1}, x_{1}^{\prime}\right]$,

$$
F_{n}\left[\wp, x_{1}\right](x) \rho\left(x_{1}\right)^{n} x_{1}^{\frac{n \beta}{\lambda^{2}}}=\mathcal{O}(1) \quad \text { as } \quad \eta \rightarrow 0
$$

and, in particular,

$$
\lim _{\eta \rightarrow 0} F_{n}\left[\wp, x_{1}\right]\left(x_{1}^{\prime}\right) \rho\left(x_{1}\right)^{n} x_{1}^{\frac{n \beta}{\lambda^{2}}}=(1+\mathcal{O}(\delta))\left(\frac{\lambda_{+} \delta^{\frac{n \beta}{\lambda^{2}}}}{n \beta}+\int_{\delta}^{2 \pi-\delta} \frac{d u}{\wp^{*}(u) f^{\delta}(u)^{n}}+\frac{\left|\lambda_{-}\right| \delta^{\frac{n \beta}{\lambda^{2}}}}{n \beta f^{\delta}(2 \pi-\delta)^{n}}\right)
$$

Hence, by (4.38) and 4.28), for any $x \in\left[x_{1}, x_{1}^{\prime}\right]$,

$$
\frac{\Sigma_{r}^{(n)}\left[\wp, x_{1}\right](x)}{\rho(x)^{n}} \rho\left(x_{1}\right)^{n} x_{1}^{\frac{n \beta}{\lambda_{+}^{2}}}=\mathcal{O}(1) \quad \text { as } \quad \eta \rightarrow 0
$$

then (4.26) follows since $\beta / \lambda_{+}^{2}=1+\theta$. From (4.39) and the definition of $f^{\delta}(x)$ it is clear that the limit

$$
\begin{array}{r}
\lim _{\eta \rightarrow 0} F_{n}\left[\wp, x_{1}\right]\left(x_{1}^{\prime}\right) \rho\left(x_{1}\right)^{n} x_{1}^{\frac{n \beta}{\lambda_{+}^{2}}}= \\
\lim _{\delta \rightarrow 0}\left(\delta^{\frac{n \beta}{\lambda^{2}}} \int_{\delta}^{2 \pi-\delta} \frac{d u}{\wp^{*}(u)} e^{-n \int_{\delta}^{u} \frac{V^{\prime}\left(u^{\prime}\right)}{\wp^{* 2}\left(u^{\prime}\right)} d u^{\prime}}+\frac{\left|\lambda_{-}\right| \delta^{n \beta\left(\lambda_{+}^{-2}+\lambda_{-}^{-2}\right)}}{n \beta} e^{-n \int_{\delta}^{2 \pi-\delta} \frac{V^{\prime}\left(u^{\prime}\right)}{\wp^{* 2}\left(u^{\prime}\right)} d u^{\prime}}\right)
\end{array}
$$

must be finite and strictly positive, thus (4.27) follows from 4.41) and 4.28).

Corollary 4.7. From 4.26 and 4.3 it follows that

$$
\sup _{x_{k} \leq x \leq x_{k}^{\prime}} \Sigma_{r}^{(n)}\left[\wp_{k}, x_{k}\right](x)=\mathcal{O}\left(\eta^{-\frac{n}{1+\theta}}\right) \quad \text { as } \quad \eta \rightarrow 0
$$

and

$$
\Sigma_{r}^{(n)}\left[\wp_{k}, x_{k}\right]\left(x_{k}^{\prime}\right)=\Theta\left(\eta^{-\frac{n}{1+\theta}}\right) \quad \text { as } \quad \eta \rightarrow 0
$$


Proof. The result follows since, from Lemma 4.2 ,

$$
\sup _{x_{k} \leq x \leq x_{k}^{\prime}} \frac{\rho_{k}(x)}{\rho_{k}\left(x_{k}\right)}=\frac{\rho_{k}\left(x_{k}^{\prime}\right)}{\rho_{k}\left(x_{k}\right)}
$$

where

$$
\frac{\rho_{k}\left(x_{k}^{\prime}\right)}{\rho_{k}\left(x_{k}\right)}=\Theta\left(\eta^{\frac{2+\theta}{1+\theta} \theta}\right)
$$

since $\frac{\beta}{\lambda_{+}^{2}}-\frac{\beta}{\lambda_{-}^{2}}=\frac{\gamma}{\beta} \sqrt{\gamma^{2}+4 \beta}=\frac{2+\theta}{1+\theta} \theta$.

Lemma 4.8. We have

$$
\sup _{x_{k} \leq x \leq x_{k}^{\prime}}\left|\Sigma_{y}^{(n)}\left[\wp_{k}, x_{k}\right](x)\right|=\mathcal{O}\left(\eta^{-n}\right) \quad \text { as } \quad \eta \rightarrow 0
$$

Proof. We prove the result for $k=1$ and omit the index 1. From 4.32, it follows that

$$
\sup _{x_{1} \leq x \leq x_{1}^{\prime}}\left|\frac{\Sigma_{y}^{(n)}\left[\wp, x_{1}\right]}{\wp(x)^{n}}\left\{\int_{x_{1}}^{x} \frac{B(u, x)^{n} d u}{\wp(u) \rho(u)^{n}}\right\}^{-1}-1\right|=o(\eta) \quad \text { as } \quad \eta \rightarrow 0
$$

with

$$
B(u, x):=\int_{u}^{x} \frac{\rho\left(u^{\prime}\right) d u^{\prime}}{\wp\left(u^{\prime}\right)^{2}}
$$

By the same techniques used in the proof of the previous Lemma it is possible to show that there exists $c>0$ such that

$$
B(u, x) \leq c \rho\left(x_{1}\right) \eta^{\frac{\beta}{\lambda^{2}}}\left[u^{-\left(1+\frac{\beta}{\lambda_{+}^{2}}\right)} \mathbf{1}_{x_{1} \leq u \leq \delta}+\mathbf{1}_{\delta \leq u \leq 2 \pi-\delta}+(2 \pi-u)^{-\left(1+\frac{\beta}{\lambda^{2}}\right)} \mathbf{1}_{2 \pi-\delta \leq u \leq x_{1}^{\prime}}\right]
$$

for any $\eta$ small enough, then, by Lemma 4.2 , there exists $c>0$ such that

$$
\frac{B(u, x)}{\rho(u)} \leq c\left[u^{-1} \mathbf{1}_{x_{1} \leq u \leq \delta}+\mathbf{1}_{\delta \leq u \leq 2 \pi-\delta}+(2 \pi-u)^{-1} \mathbf{1}_{2 \pi-\delta \leq u \leq x_{1}^{\prime}}\right]
$$

hence

$$
\sup _{x_{1} \leq x \leq x_{1}^{\prime}} \int_{x_{1}}^{x} \frac{B(u, x)^{n} d u}{\wp(u) \rho(u)^{n}}=\mathcal{O}\left(\eta^{-n}\right) \quad \text { as } \quad \eta \rightarrow 0
$$

thus 4.47) follows from 4.48 and 4.52.

\section{Estimates of the variances}

We recall that $\left(X_{k}(t), P_{k}(t)\right), T_{k} \leq t \leq S_{k}$ is the solution of the problem

$$
\begin{cases}\dot{X}_{k}=P_{k} & X_{k}\left(T_{k}\right)=x_{k} \\ \dot{P}_{k}=-\gamma P_{k}-V^{\prime}\left(X_{k}\right) & P_{k}\left(T_{k}\right)=p_{k}\end{cases}
$$

$\wp_{k}(x)$ is the related orbit in $[2(k-1) \pi, 2 k \pi]$, i.e. the path such that $\wp_{k}\left(X_{k}(t)\right)=P_{k}(t)$ for $T_{k} \leq t \leq S_{k}$ and $\omega_{k}(t)=\frac{d}{d x} \wp_{k}\left(X_{k}(t)\right)$.

In this Section we provide some estimates on the variances of the processes $\bar{y}_{k}(t)$ and $\bar{r}_{k}(t)$. The two following Lemmas follow directly from their definitions in 2.15) and 2.14. 
Lemma 5.1. Let

$$
\sigma_{r}^{2}\left(T_{k}, t\right):=\epsilon^{2} \int_{T_{k}}^{t} e^{-2 \int_{s}^{t}\left(\omega_{k}\left(s^{\prime}\right)+\gamma\right) d s^{\prime}} d s
$$

then

$$
\bar{r}_{k}(t) \mid T_{k} \sim \operatorname{Gauss}\left(0, \sigma_{r}\left(T_{k}, t\right)\right), \quad t \geq T_{k}
$$

Lemma 5.2. Let

$$
\sigma_{y}^{2}\left(t, T_{k}\right):=\epsilon^{2} \int_{T_{k}}^{t} e^{2 \int_{s}^{t} \omega_{k}\left(s^{\prime \prime}\right) d s^{\prime \prime}}\left[\int_{s}^{t} e^{-\int_{s}^{s^{\prime}}\left(2 \omega_{k}\left(s^{\prime \prime}\right)+\gamma\right) d s^{\prime \prime}} d s^{\prime}\right]^{2} d s
$$

then

$$
\bar{y}_{k}(t) \mid T_{k} \sim \text { Gauss }\left(0, \sigma_{y}\left(T_{k}, t\right)\right), \quad t \geq T_{k}
$$

Let $\eta_{\epsilon}>0$ be as in Section 2, we recall that

$$
\mathcal{H}_{k}^{\xi}=\left\{(x, p): p=\eta_{\epsilon}+\lambda_{-}(x-2(k-1) \pi),\left|p-\lambda_{+}(x-2(k-1) \pi)\right| \leq \bar{\sigma}_{\epsilon} \epsilon^{-\xi}\right\}, \quad \text { for } \quad k \geq 1
$$

and $\mathcal{H}_{0}=\left\{\left(-\pi, \wp_{0}^{*}(-\pi)\right)\right\}$, and define the stopping time

$$
\bar{S}_{k}:=\inf \left\{t \geq T_{k}: X_{k}(t) \geq 2 k \pi-\frac{\eta_{\epsilon}}{2\left(\lambda_{+}-\lambda_{-}\right)}\right\}
$$

We denote by $x_{k}^{\prime}:=X\left(\bar{S}_{k}\right)$, then

$$
2 k \pi-x_{k}^{\prime}=\frac{\eta_{\epsilon}}{2\left(\lambda_{+}-\lambda_{-}\right)}
$$

and $x_{k} \leq X_{k}(t) \leq x_{k}^{\prime}$ for $T_{k} \leq t \leq \bar{S}_{k}$.

In the following Lemma we prove that, if $\left(x_{k}, p_{k}\right) \in \mathcal{H}_{k}^{\xi}$, then $x_{k}, x_{k}^{\prime}$ and $\wp_{k}(\cdot)$ satisfy the conditions (4.3) and (4.4) in $[2(k-1) \pi, 2 k \pi]$ in the following sense.

Lemma 5.3. Let $\left(x_{k}, p_{k}\right) \in \mathcal{H}_{k}^{\xi}$, with $\xi$ small enough, then

$$
x_{k}-2(k-1) \pi=\Theta\left(\eta_{\epsilon}\right) \quad \text { and } \quad 2 k \pi-x_{k}^{\prime}=\Theta\left(\eta_{\epsilon}\right) \quad \text { as } \quad \epsilon \rightarrow 0
$$

moreover

$$
\left|\wp_{k}\left(x_{k}\right)-\wp_{k}^{*}\left(x_{k}\right)\right|=o\left(\eta_{\epsilon}\right) \quad \text { as } \quad \epsilon \rightarrow 0
$$

Proof. (5.7) follows directly from the definition of $\mathcal{H}_{k}^{\xi}$ and from (5.6), whereas (5.8) is verified since

$$
\begin{aligned}
& \left|\wp_{k}\left(x_{k}\right)-\wp_{k}^{*}\left(x_{k}\right)\right|=\left|p_{k}-\wp_{k}^{*}\left(x_{k}\right)\right| \\
& \leq\left|p_{k}-\lambda_{+}\left(x_{k}-2(k-1) \pi\right)\right|+\left|\lambda_{+}\left(x_{k}-2(k-1) \pi\right)-\wp_{k}^{*}\left(x_{k}\right)\right| \\
& \leq \bar{\sigma}_{\epsilon} \epsilon^{-\xi}+c \eta_{\epsilon}^{2} \leq 2 \bar{\sigma}_{\epsilon} \epsilon^{-\xi}
\end{aligned}
$$

where the last inequality follows from the definition of $\mathcal{H}_{k}^{\xi},(4.1)$ and the left hand side of (5.7). Hence 5.8 holds if $\xi$ is small enough, since, from (2.22), $\bar{\sigma}_{\epsilon}=o\left(\eta_{\epsilon}\right)$ as $\epsilon \rightarrow 0$.

Lemma 5.4. Let $\left(x_{k}, p_{k}\right) \in \mathcal{H}_{k}^{\xi}$, with $\xi$ small enough, then

$$
\wp_{k}\left(x_{k}^{\prime}\right)=\lambda_{-}\left(x_{k}^{\prime}-2 k \pi\right)+\mathcal{O}\left(\tilde{\sigma}_{\epsilon} \epsilon^{-\xi}\right) \quad \text { as } \quad \epsilon \rightarrow 0
$$


Proof. From 4.20 and $(5.9)$ we have

$$
\left|\wp_{k}\left(x_{k}^{\prime}\right)-\wp_{k}^{*}\left(x_{k}^{\prime}\right)\right| \leq c \eta_{\epsilon}^{\frac{2+\theta}{1+\theta} \theta} \bar{\sigma}_{\epsilon} \epsilon^{-\xi}=\mathcal{O}\left(\tilde{\sigma}_{\epsilon} \epsilon^{-\xi}\right) \quad \text { as } \quad \epsilon \rightarrow 0
$$

then the result follows from 4.2 since, from $(2.22), \eta_{\epsilon}^{2}=o\left(\tilde{\sigma}_{\epsilon}\right)$ as $\epsilon \rightarrow 0$.

Remark 5.5. As a consequence of Lemma 5.3 and the considerations done in Section 4.1, $\wp_{k}(\cdot)$ is well defined in $\left[x_{k}, x_{k}^{\prime}\right]$.

Proposition 5.6. Let $\left(x_{k}, p_{k}\right) \in \mathcal{H}_{k}^{\xi}$, $\xi$ small enough, then

$$
\sup _{T_{k} \leq t \leq \bar{S}_{k}} \sigma_{r}^{2}\left(T_{k}, t\right)=\mathcal{O}\left(\sigma_{\epsilon}^{2}\right) \quad \text { as } \quad \epsilon \rightarrow 0 \quad \mathbf{P}_{T_{k}, x_{k}, p_{k}} \text { a.s. }
$$

Proof. We have

$$
\sigma_{r}^{2}\left(T_{k}, t\right)=\epsilon^{2} \int_{x_{k}}^{X_{k}(t)} e^{-2 \int_{u}^{X_{k}(t)} \frac{\wp_{k}^{\prime}\left(u^{\prime}\right)+\gamma}{\wp_{k}\left(u^{\prime}\right)} d u^{\prime}} \frac{d u}{\wp_{k}(u)}=\epsilon^{2} \Sigma_{r}^{(2)}\left[\wp_{k}, x_{k}\right]\left(X_{k}(t)\right)
$$

where the second identity follows from 1.5 , then

$$
\sup _{T_{k} \leq t \leq \bar{S}_{k}} \sigma_{r}^{2}\left(T_{k}, t\right)=\epsilon^{2} \sup _{x_{k} \leq x \leq x_{k}^{\prime}} \Sigma_{r}^{(2)}\left[\wp_{k}, x_{k}\right](x)=\epsilon^{2} \mathcal{O}\left(\eta_{\epsilon}^{-\frac{2}{1+\theta}}\right)
$$

where the last equivalence follows from 4.43 . Then 5.12 follows from the definition of $\sigma_{\epsilon}$ in (2.19.

Proposition 5.7. Let $\left(x_{k}, p_{k}\right) \in \mathcal{H}_{k}^{\xi}$, $\xi$ small enough, then

$$
\sup _{T_{k} \leq t \leq \bar{S}_{k}} \sigma_{y}^{2}\left(T_{k}, t\right)=\mathcal{O}\left(\epsilon^{2} \eta_{\epsilon}^{-2}\right) \quad \mathbf{P}_{T_{k}, x_{k}, p_{k}} \text { a.s. }
$$

Proof. We have

$$
\begin{aligned}
& \sigma_{y}^{2}\left(T_{k}, t\right)=\epsilon^{2} \int_{T_{k}}^{t} e^{2 \int_{X_{k}(s)}^{X_{k}(t)} \frac{\wp_{k}^{\prime}\left(u^{\prime}\right)}{\wp_{k}\left(u^{\prime}\right)} d u^{\prime}}\left[\int_{s}^{t} e^{-\int_{X_{k}(s)}^{X_{k}\left(s^{\prime}\right)} \frac{2 \wp_{k}^{\prime}\left(u^{\prime}\right)+\gamma}{\wp_{k}\left(u^{\prime}\right)} d u^{\prime}} d s^{\prime}\right]^{2} d s \\
& =\wp_{k}^{2}\left(X_{k}(t)\right) \int_{x_{k}}^{X_{k}(t)} \wp_{k}(u)\left[\int_{u}^{X_{k}(t)} \frac{d u^{\prime}}{\wp_{k}^{3}\left(u^{\prime}\right)} e^{-\int_{u}^{u^{\prime}} \frac{\gamma}{\wp_{k}\left(u^{\prime \prime}\right)} d u^{\prime \prime}}\right]^{2} d u \\
& =\epsilon^{2} \Sigma_{y}^{(2)}\left[\wp_{k}, x_{k}\right]\left(X_{k}(t)\right)
\end{aligned}
$$

thus

$$
\sup _{T_{k} \leq t \leq \bar{S}_{k}} \sigma_{y}^{2}\left(T_{k}, t\right)=\epsilon^{2} \sup _{x_{k} \leq x \leq x_{k}^{\prime}} \Sigma_{y}^{(2)}\left[\wp_{k}, x_{k}\right](x)=\epsilon^{2} \mathcal{O}\left(\eta_{\epsilon}^{-2}\right)
$$

where the last equivalence follows from 4.47). 
Lemma 5.8. Let $\left(x_{k}, p_{k}\right) \in \mathcal{H}_{k}^{\xi}, \xi$ small enough, then there exists $C>0$ such that

$$
\sup _{\epsilon<1} \sup _{T_{k} \leq t \leq \bar{S}_{k}}\left|\omega_{k}(t)\right| \leq C
$$

Proof. It is a direct consequence of Lemma 4.4 since

$$
\sup _{T_{k} \leq t \leq \bar{S}_{k}}\left|\omega_{k}(t)\right|=\sup _{x_{k} \leq x \leq x_{k}^{\prime}}\left|\frac{d}{d x} \wp_{k}(x)\right|
$$

Lemma 5.9. Let

$$
H_{r}\left(T_{k}, t\right):=\int_{T_{k}}^{t} e^{-\int_{s}^{t}\left(\omega_{k}\left(s^{\prime}\right)+\gamma\right) d s^{\prime}} d s
$$

and

$$
H_{y}\left(t, T_{k}\right):=\int_{T_{k}}^{t} e^{\int_{s}^{t} \omega_{k}\left(s^{\prime \prime}\right) d s^{\prime \prime}}\left[\int_{s}^{t} e^{-\int_{s}^{s^{\prime}}\left(2 \omega_{k}\left(s^{\prime \prime}\right)+\gamma\right) d s^{\prime \prime}} d s^{\prime}\right] d s
$$

then, for $\left(x_{k}, p_{k}\right) \in \mathcal{H}_{k}^{\xi}$, $\xi$ small enough, we have

$$
\sup _{T_{k} \leq t \leq \bar{S}_{k}} H_{r}\left(T_{k}, t\right)=\mathcal{O}\left(\eta_{\epsilon}^{-\frac{1}{1+\theta}}\right)
$$

and

$$
\sup _{T_{k} \leq t \leq \bar{S}_{k}} H_{y}\left(T_{k}, t\right)=\mathcal{O}\left(\eta_{\epsilon}^{-1}\right)
$$

Proof. It follows directly from 4.43 and 4.47 since

$$
H_{r}\left(T_{k}, t\right)=\Sigma_{r}^{(1)}\left[\wp_{k}, x_{k}\right]\left(X_{k}(t)\right) \quad \text { and } \quad H_{y}\left(T_{k}, t\right)=\Sigma_{y}^{(1)}\left[\wp_{k}, x_{k}\right]\left(X_{k}(t)\right)
$$

\section{$6 \quad$ Estimate of Errors}

In this Section we prove Propositions 3.6, 3.7, 3.8, 3.9 and 3.10

\section{Errors in the stable interval}

We denote by $\mathbf{P}_{T_{k}, x_{k}, p_{k}}$ the law of $(x(t), p(t))$ given $x\left(T_{k}\right)=x_{k}, p\left(T_{k}\right)=p_{k}$. In this first part of the Section we will prove the following Proposition.

Proposition 6.1. There exists $C>0$ such that, for any $\zeta>0, \xi, \epsilon$ small enough,

$$
\begin{gathered}
\mathbf{1}_{\left(x_{k}, p_{k}\right) \in \mathcal{H}_{k}^{\xi}} \mathbf{P}_{T_{k}, x_{k}, p_{k}}\left\{\sup _{T_{k} \leq t \leq S_{k}}\left|y_{k}(t)\right| \geq \frac{\epsilon}{\eta_{\epsilon}} \epsilon^{-\zeta}\right\} \leq e^{-C \epsilon^{-2 \zeta}} \\
\mathbf{1}_{\left(x_{k}, p_{k}\right) \in \mathcal{H}_{k}^{\xi}} \mathbf{P}_{T_{k}, x_{k}, p_{k}}\left\{\sup _{T_{k} \leq t \leq S_{k}}\left|\mathcal{R}_{k}(t)\right| \geq \frac{\epsilon \sigma_{\epsilon}}{\eta_{\epsilon}^{2}} \epsilon^{-2 \zeta}\right\} \leq e^{-C \epsilon^{-2 \zeta}}
\end{gathered}
$$

and

$$
\mathbf{1}_{\left(x_{k}, p_{k}\right) \in \mathcal{H}_{k}^{\xi}} \mathbf{P}_{T_{k}, x_{k}, p_{k}}\left\{\sup _{T_{k} \leq t \leq S_{k}}\left|r_{k}(t)\right| \geq \sigma_{\epsilon} \epsilon^{-\zeta}\right\} \leq e^{-C \epsilon^{-2 \zeta}}
$$


Lemma 6.2. There exists $C>0$ such that, for any $\zeta>0, \xi, \epsilon$ small enough,

$$
\mathbf{1}_{\left(x_{k}, p_{k}\right) \in \mathcal{H}_{k}^{\xi}} \mathbf{P}_{T_{k}, x_{k}, p_{k}}\left\{\sup _{T_{k} \leq t \leq \bar{S}_{k}}\left|y_{k}(t)\right| \geq \frac{\epsilon}{\eta_{\epsilon}} \epsilon^{-\zeta}\right\} \leq e^{-C \epsilon^{-2 \zeta}}
$$

Proof. Suppose that $\left(x_{k}, p_{k}\right) \in \mathcal{H}_{k}^{\xi}$ for some $\xi>0$ small enough. Let us fix $\zeta>0$, then, from Lemma 5.2. (7.4) and (5.15) it follows that there exists $C>0$ such that, for any $\epsilon$ small enough,

$$
\mathbf{P}_{T_{k}, x_{k}, p_{k}}\left\{\sup _{T_{k} \leq t \leq \bar{S}_{k}}\left|\bar{y}_{k}(t)\right| \geq \frac{\epsilon}{2 \eta_{\epsilon}} \epsilon^{-\zeta}\right\} \leq e^{-C \epsilon^{-2 \zeta}}
$$

We recall that $y_{k}(t)=\bar{y}_{k}(t)+\mathcal{Y}_{k}(t)$ with

$$
\mathcal{Y}_{k}(t) \leq H_{y}\left(T_{k}, t\right) \cdot \sup _{T_{k} \leq s \leq \overline{S_{k}}} \varphi\left(X_{k}(s), y_{k}(s)\right) \quad \text { for } \quad T_{k} \leq t \leq \bar{S}_{k}
$$

(see 2.16 and (5.25). From (2.7), we know that $\varphi(x, y)=\mathcal{O}\left(y^{2}\right)$ for small $|y|$, then if we define the stopping time $\Upsilon_{k}^{\zeta}:=\inf \left\{t \geq T_{k}:\left|y_{k}(t)\right| \geq \epsilon^{1-\zeta} \eta_{\epsilon}^{-1}\right\}$, then there exists $c>0$ such that

$$
\sup _{T_{k} \leq s \leq \Upsilon_{k}^{\zeta} \wedge \bar{S}_{k}}\left|\varphi\left(X_{k}(s), y_{k}(s)\right)\right| \leq c \frac{\epsilon^{2(1-\zeta)}}{\eta_{\epsilon}^{2}}
$$

thus, from (6.6) and 5.25 we have

$$
\left|\mathcal{Y}_{k}(t)\right| \leq c \frac{\epsilon^{2(1-\zeta)}}{\eta_{\epsilon}^{3}} \quad \text { for any } \quad T_{k} \leq t \leq \Upsilon_{k}^{\zeta} \wedge \bar{S}_{k} .
$$

Thus, since $\left|y_{k}(t)\right| \leq\left|\bar{y}_{k}(t)\right|+\left|\mathcal{Y}_{k}(t)\right|$, by 6.5) and (6.8), with $\mathbf{P}_{T_{k}, x_{k}, p_{k}}$-probability greater than $1-2 e^{-C \epsilon^{-2 \zeta}}$,

$$
\sup _{T_{k} \leq t \leq \Upsilon_{k}^{\zeta} \wedge \bar{S}_{k}}\left|y_{k}(t)\right| \leq \frac{\epsilon^{1-\zeta}}{2 \eta_{\epsilon}}+c \frac{\epsilon^{2(1-\zeta)}}{\eta_{\epsilon}^{3}}<\frac{\epsilon^{1-\zeta}}{\eta_{\epsilon}},
$$

hence, in particular, with the same probability, $\bar{S}_{k}<\Upsilon_{k}^{\zeta}$, then $(6.4)$ follows.

Lemma 6.3. There exists $C>0$ such that, for any $\zeta>0, \xi, \epsilon$ small enough,

$$
\mathbf{1}_{\left(x_{k}, p_{k}\right) \in \mathcal{H}_{k}^{\xi}} \mathbf{P}_{T_{k}, x_{k}, p_{k}}\left\{\sup _{T_{k} \leq t \leq \bar{S}_{k}}\left|\mathcal{R}_{k}(t)\right| \geq \frac{\epsilon \sigma_{\epsilon}}{\eta_{\epsilon}^{2}} \epsilon^{-2 \zeta}\right\} \leq e^{-C \epsilon^{-2 \zeta}}
$$

Proof. Suppose that $\left(x_{k}, p_{k}\right) \in \mathcal{H}_{k}^{\xi}$ for some $\xi$ small enough. From 2.17) we know that

$$
\mathcal{R}_{k}(t)=H_{r}\left(T_{k}, t\right) \cdot \sup _{T_{k} \leq t \leq \bar{S}_{k}} \varphi\left(X_{k}(s), y_{k}(s)\right) d s
$$

thus, by Lemma 6.2, we know that there exists $c>0$ such that

$$
\sup _{T_{k} \leq s \leq \bar{S}_{k}}\left|\varphi\left(X_{k}(s), y_{k}(s)\right)\right| \leq c \frac{\epsilon^{2(1-\zeta)}}{\eta_{\epsilon}^{2}}
$$


with $\mathbf{P}_{T_{k}, x_{k}, p_{k}}$-probability greater than $1-e^{-C \epsilon^{-2 \zeta}}$, then, from (6.10), 6.11) and (5.24) it follows that

$$
\sup _{T_{k} \leq t \leq \bar{S}_{k}}\left|\mathcal{R}_{k}(t)\right| \leq c \frac{\epsilon^{2(1-\zeta)}}{\eta_{\epsilon}^{2}} \eta_{\epsilon}^{-\frac{1}{1+\theta}}
$$

with $\mathbf{P}_{T_{k}, x_{k}, p_{k}}$-probability greater than $1-e^{-C \epsilon^{-2 \zeta}}$, hence 6.9 follows from the definition of $\sigma_{\epsilon}$.

Lemma 6.4. There exists $C>0$ such that, for any $\zeta>0, \xi, \epsilon$ small enough,

$$
\mathbf{1}_{\left(x_{k}, p_{k}\right) \in \mathcal{H}_{k}^{\xi}} \mathbf{P}_{T_{k}, x_{k}, p_{k}}\left\{\sup _{T_{k} \leq t \leq \bar{S}_{k}}\left|r_{k}(t)\right| \geq \sigma_{\epsilon} \epsilon^{-\zeta}\right\} \leq e^{-C \epsilon^{-2 \zeta}}
$$

Proof. Assume $\left(x_{k}, p_{k}\right) \in \mathcal{H}_{k}^{\xi}$, with $\xi$ small enough. We recall that $r_{k}(t)=\bar{r}_{k}(t)+\mathcal{R}_{k}(t)$. From Lemma 5.1. (7.4) and 5.12, there exists $C>0$ such that, for any $\zeta>0, \epsilon$ small enough,

$$
\mathbf{P}_{T_{k}, x_{k}, p_{k}}\left\{\sup _{T_{k} \leq t \leq \bar{S}_{k}}\left|\bar{r}_{k}(t)\right| \geq \frac{\sigma_{\epsilon}}{2} \epsilon^{-\zeta}\right\} \leq e^{-C \epsilon^{-2 \zeta}}
$$

hence the result descends from 6.14 and Lemma 6.3, since, from 2.22, $\epsilon=o\left(\eta_{\epsilon}^{2}\right)$.

Corollary 6.5. There exists $C>0$ such that, for any $\zeta>0, \xi, \epsilon$ small enough,

$$
\mathbf{1}_{\left(x_{k}, p_{k}\right) \in \mathcal{H}_{k}^{\xi}} \mathbf{P}_{T_{k}, x_{k}, p_{k}}\left\{\sup _{T_{k} \leq t \leq \bar{S}_{k}}\left|q_{k}(t)\right| \geq \frac{\epsilon}{\eta_{\epsilon}} \epsilon^{-\zeta}\right\} \leq e^{-C \epsilon^{-2 \zeta}}
$$

Proof. We recall that $q_{k}(t)=r_{k}(t)+\omega_{k}(t) y_{k}(t)$, then 6.15) easily follows from Lemma 6.2. Lemma 6.4 and Lemma 5.8 .

Lemma 6.6. Let $\bar{S}_{k}$ be the stopping time defined in 5.5) and

$$
\tilde{S}_{k}:=\inf \left\{t \geq T_{k}: X_{k}(t) \geq 2 k \pi-\frac{2 \eta_{\epsilon}}{\left(\lambda_{+}-\lambda_{-}\right)}\right\}
$$

then there exists $C>0$ such that, for any $\zeta>0, \xi, \epsilon$ small enough,

$$
\mathbf{1}_{\left(x_{k}, p_{k}\right) \in \mathcal{H}_{k}^{\xi}} \mathbf{P}_{T_{k}, x_{k}, p_{k}}\left\{\tilde{S}_{k} \leq S_{k} \leq \bar{S}_{k}\right\} \geq 1-e^{-C \epsilon^{-2 \zeta}}
$$

Proof. We only show that

$$
\mathbf{1}_{\left(x_{k}, p_{k}\right) \in \mathcal{H}_{k}^{\xi}} \mathbf{P}_{T_{k}, x_{k}, p_{k}}\left\{S_{k} \leq \bar{S}_{k}\right\} \geq 1-e^{-C \epsilon^{-2 \zeta}}
$$

since the arguments for the estimate of $\mathbf{P}_{T_{k}, x_{k}, p_{k}}\left\{\tilde{S}_{k} \leq S_{k}\right\}$ are specular. Assume $\left(x_{k}, p_{k}\right) \in \mathcal{H}_{k}^{\xi}$, for some $\xi$ small enough. We have

$$
\mathbf{P}_{T_{k}, x_{k}, p_{k}}\left\{S_{k} \leq \bar{S}_{k}\right\} \geq \mathbf{P}_{T_{k}, x_{k}, p_{k}}\left\{v_{k}\left(\bar{S}_{k}\right) \leq \eta_{\epsilon}\right\}
$$


where

$$
v_{k}(t)=P_{k}(t)-\lambda_{+}\left(X_{k}(t)-2 k \pi\right)+q_{k}(t)-\lambda_{+} y_{k}(t) .
$$

Recalling the definition of $\bar{S}_{k}$ and $x_{k}^{\prime}$ in $(5.5)$, we have

$$
v_{k}\left(\bar{S}_{k}\right)=\wp_{k}\left(x_{k}^{\prime}\right)-\lambda_{+}\left(x_{k}^{\prime}-2 k \pi\right)+q_{k}\left(\bar{S}_{k}\right)-\lambda_{+} y_{k}\left(\bar{S}_{k}\right) .
$$

thus, from Lemma 5.4 we have

$$
\left|v_{k}\left(\bar{S}_{k}\right)-\frac{\eta_{\epsilon}}{2}-\left(q_{k}\left(\bar{S}_{k}\right)-\lambda_{+} y_{k}\left(\bar{S}_{k}\right)\right)\right| \leq c \tilde{\sigma}_{\epsilon} \epsilon^{-\xi}
$$

for some $c>0$. By Lemma 6.2 and Corollary 6.5 , there exists $C>0$ such that, for any $\zeta, \epsilon$ small enough,

$$
\mathbf{P}_{T_{k}, x_{k}, p_{k}}\left\{\left|q_{k}\left(\bar{S}_{k}\right)-\lambda_{+} y_{k}\left(\bar{S}_{k}\right)\right| \geq \frac{\epsilon}{\eta_{\epsilon}} \epsilon^{-\zeta}\right\} \leq \epsilon^{-C \epsilon^{-2 \zeta}}
$$

From 2.22 we know that $\tilde{\sigma}_{\epsilon} \epsilon^{-\xi}=o\left(\eta_{\epsilon}\right)$ and $\epsilon^{1-\xi}=o\left(\eta_{\epsilon}^{2}\right)$, for $\xi$ small enough, thus, from 6.22 and (6.23) it follows that

$$
\mathbf{P}_{T_{k}, x_{k}, p_{k}}\left\{v_{k}\left(\bar{S}_{k}\right)>\eta_{\epsilon}\right\} \leq \epsilon^{-C \epsilon^{-2 \zeta}}
$$

then 6.18) follows from 6.19) and 6.24.

Proof of Proposition 6.1. It directly follows from $(6.4),(6.9), 6.13)$ and Lemma 6.6

\section{Errors in the critical interval}

For any $\xi>0$ we recall that

$$
\mathcal{K}_{k}^{\xi}=\left\{(x, p): p-\lambda_{+}(x-2 k \pi)=\eta_{\epsilon},\left|p-\lambda_{-}(x-2 k \pi)\right| \leq \sigma_{\epsilon} \epsilon^{-\xi}\right\} .
$$

and denote by $\mathbf{P}_{S_{k}, \bar{x}_{k}, \bar{p}_{k}}$ the law of $(x(t), p(t))$ given $x\left(S_{k}\right)=\bar{x}_{k}, p\left(S_{k}\right)=\bar{p}_{k}$. We will prove the following result.

Proposition 6.7. We have

$$
\sup _{S_{k} \leq t \leq T_{k+1}}\left|z_{k}(t)\right| \leq \eta_{\epsilon} \quad \mathbf{P}_{S_{k}, \bar{x}_{k}, \bar{p}_{k}} \text { a.s. }
$$

moreover there exists $C>0$ such that, for any $\delta>0, \zeta, \epsilon$ small enough,

$$
\mathbf{P}_{S_{k}, \bar{x}_{k}, \bar{p}_{k}}\left\{\sup _{S_{k} \leq t \leq T_{k+1}}\left|v_{k}(t)\right| \geq(1+\delta) \eta_{\epsilon}\right\} \leq e^{-C \epsilon^{-2 \zeta}}
$$

for any $\left(\bar{x}_{k}, \bar{p}_{k}\right) \in \mathbb{R}^{2}$.

Lemma 6.8. There exists $C>0$ such that, for any $\delta, \zeta>0, \epsilon$ small enough,

$$
\mathbf{P}_{S_{k}, \bar{x}_{k}, \bar{p}_{k}}\left\{\sup _{t \geq S_{k}}\left|\bar{v}_{k}(t)\right| \leq(1+\delta) \eta_{\epsilon}\right\} \geq 1-e^{-C \epsilon^{-2 \zeta}}
$$

for any $\left(\bar{x}_{k}, \bar{p}_{k}\right) \in \mathbb{R}^{2}$. 
Proof. From Lemma 3.1 we know that the probability law of $\bar{v}_{k}\left(S_{k}+t\right)-\eta_{\epsilon} e^{\lambda_{-}}{ }^{t}$ is a centered Gaussian of variance $\sigma_{v}^{2}(t)$, independently on the initial condition $\left(\bar{x}_{k}, \bar{p}_{k}\right)$ at time $S_{k}$. By (3.1) we have

$$
\sup _{t \geq 0} \sigma_{v}^{2}(t) \leq \frac{\epsilon^{2}}{2\left|\lambda_{-}\right|}
$$

thus, by (7.4), there exists $C>0$ such that,

$$
\mathbf{P}_{S_{k}, \bar{x}_{k}, \bar{p}_{k}}\left\{\sup _{t \geq 0}\left|\bar{v}_{k}\left(S_{k}+t\right)-\eta_{\epsilon} e^{\lambda_{-} t}\right| \geq \epsilon^{1-\zeta}\right\} \leq e^{-C \epsilon^{-2 \zeta}}
$$

for any $\zeta, \delta>0, \epsilon$ small enough, $\left(\bar{x}_{k}, \bar{p}_{k}\right) \in \mathbb{R}^{2}$, then 6.28 follows since, from $2.22, \epsilon^{1-\zeta}=o\left(\eta_{\epsilon}\right)$.

Lemma 6.9. There exists $C>0$ such that, for any $\xi>0, \zeta, \epsilon$ small enough,

$$
\mathbf{1}_{\left(\bar{x}_{k}, \bar{p}_{k}\right) \in \mathcal{K}_{k}^{\xi}} \mathbf{P}_{S_{k}, \bar{x}_{k}, \bar{p}_{k}}\left\{\sup _{t \geq S_{k}}\left|\bar{z}_{k}(t)\right| e^{-\lambda_{+}\left(t-S_{k}\right)} \geq \sigma_{\epsilon} \epsilon^{-\xi}\right\} \leq e^{-C \epsilon^{-2 \zeta}}
$$

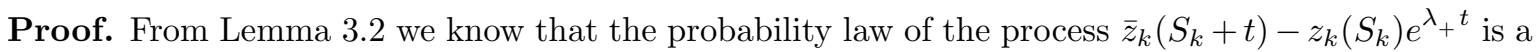
centered Gaussian of variance $\sigma_{z}^{2}(t)$ independent of the values $\left(\bar{x}_{k}, \bar{p}_{k}\right)$ at time $S_{k}$. From the formula in 3.2 we have

$$
\sup _{t \geq 0} \sigma_{z}^{2}(t) e^{-2 \lambda_{+} t} \leq \frac{\epsilon^{2}}{2 \lambda_{+}}
$$

thus, by 7.4 , there exists $C>0$ such that, for any $\zeta>0, \epsilon$ small enough, $\left(\bar{x}_{k}, \bar{p}_{k}\right) \in \mathbb{R}^{2}$,

$$
\mathbf{P}_{S_{k}, \bar{x}_{k}, \bar{p}_{k}}\left\{\sup _{t \geq 0}\left|\bar{z}_{k}\left(S_{k}+t\right) e^{-\lambda_{+} t}-z_{k}\left(S_{k}\right)\right| \geq \epsilon^{1-\zeta}\right\} \leq e^{-C \epsilon^{-2 \zeta}}
$$

then, with $\mathbf{P}_{S_{k}, \bar{x}_{k}, \bar{p}_{k}}$-probability larger than $1-e^{-C \epsilon^{-2 \zeta}}$

$$
\left|\bar{z}_{k}(t)\right| e^{-\lambda_{+}\left(t-S_{k}\right)} \leq\left|z_{k}\left(S_{k}\right)\right|+\epsilon^{1-\zeta} \quad \forall t \geq S_{k} .
$$

Let us assume $\left(\bar{x}_{k}, \bar{p}_{k}\right) \in \mathcal{K}_{k}^{\xi}$, for some $\xi>0$, hence 6.31 follows since $\left|z_{k}\left(S_{k}\right)\right|=\left|p-\lambda_{-}(x-2 k \pi)\right| \leq$ $\sigma_{\epsilon} \epsilon^{-\xi}$ and since, from $2.22, \epsilon^{1-\zeta}=o\left(\sigma_{\epsilon} \epsilon^{-\xi}\right)$, for $\zeta$ small enough.

Proof of Propositions 6.7, 3.8 and 3.9, 6.26 follows directly from the definitions of $T_{k+1}$ and $\mathcal{K}_{k}^{\xi}$, since $\sigma_{\epsilon} \epsilon^{-\xi}=o\left(\eta_{\epsilon}\right)$ for $\xi$ small enough.

Assume $\left(\bar{x}_{k}, \bar{p}_{k}\right) \in \mathcal{K}_{k}^{\xi}$, thus $|x-2 k \pi|<3 \eta_{\epsilon} /\left(\lambda_{+}-\lambda_{-}\right)$. Consider the stopping time

$$
\Gamma_{k}:=\inf \left\{t \geq S_{k}:|x(t)-2 k \pi| \geq \frac{3 \eta_{\epsilon}}{\lambda_{+}-\lambda_{-}}\right\}
$$

then, since $\psi_{k}(x)=\mathcal{O}\left((x-2 k \pi)^{2}\right)$ for small $(x-2 k \pi)$, by 2.4 there exists $c>0$ such that, for any $S_{k} \leq t \leq \Gamma_{k} \wedge T_{k+1}$,

$$
\left|\mathcal{V}_{k}(t)\right| \leq c \eta_{\epsilon}^{2} e^{\lambda_{-} t} \int_{S_{k}}^{t} e^{-\lambda_{-} s} d s \leq c \eta_{\epsilon}^{2}
$$


and

$$
\left|\mathcal{Z}_{k}(t)\right| \leq c \eta_{\epsilon}^{2} e^{\lambda_{+} t} \int_{S_{k}}^{t} e^{-\lambda_{+} s} d s \leq c \eta_{\epsilon}^{2} e^{\lambda_{+}\left(t-S_{k}\right)}
$$

then

$$
\sup _{S_{k} \leq t \leq \Gamma_{k} \wedge T_{k+1}}\left|\mathcal{V}_{k}(t)\right| \leq c^{\prime} \eta_{\epsilon}^{2} \quad \text { and } \quad \sup _{S_{k} \leq t \leq \Gamma_{k} \wedge T_{k+1}}\left|\mathcal{Z}_{k}(t)\right| e^{-\lambda_{+}\left(t-S_{k}\right)} \leq c^{\prime} \eta_{\epsilon}^{2}
$$

for suitable $c^{\prime}>0$. Recalling that $z_{k}(t)=\bar{z}_{k}(t)+\mathcal{Z}_{k}(t)$, since $\eta_{\epsilon}^{2}=o\left(\sigma_{\epsilon}\right)$, it follows from 6.31) and the right hand side of (6.37) that, for any $\xi>0, \zeta, \epsilon$ small enough,

$$
\mathbf{1}_{\left(\bar{x}_{k}, \bar{p}_{k}\right) \in \mathcal{K}_{k}^{\xi}} \mathbf{P}_{S_{k}, \bar{x}_{k}, \bar{p}_{k}}\left\{\sup _{S_{k} \leq t \leq \Gamma_{k} \wedge T_{k+1}}\left|z_{k}(t)\right| e^{-\lambda_{+}\left(t-S_{k}\right)} \geq \sigma_{\epsilon} \epsilon^{-\xi}\right\} \leq e^{-C \epsilon^{-2 \zeta}}
$$

for a suitable $C>0$.

On the other hand we have $v_{k}(t)=\bar{v}_{k}(t)+\mathcal{V}_{k}(t)$, then, by Lemma 6.8 and 6.37), there exists $C>0$ such that, for any $\left(\bar{x}_{k}, \bar{p}_{k}\right) \in \mathbb{R}^{2}, \zeta, \delta>0, \epsilon$ small enough,

$$
\mathbf{P}_{S_{k}, \bar{x}_{k}, \bar{p}_{k}}\left\{\sup _{S_{k} \leq t \leq \Gamma_{k} \wedge T_{k+1}}\left|v_{k}(t)\right| \geq \eta_{\epsilon}(1+\delta)\right\} \leq e^{-C \epsilon^{-2 \zeta}}
$$

We recall that $x(t)-2 k \pi=\left(z_{k}(t)-v_{k}(t)\right) /\left(\lambda_{+}-\lambda_{-}\right)$, thus from 6.26) and 6.39) it follows that

$$
\mathbf{P}_{S_{k}, \bar{x}_{k}, \bar{p}_{k}}\left\{\Gamma_{k}>T_{k+1}\right\} \geq \mathbf{P}_{S_{k}, \bar{x}_{k}, \bar{p}_{k}}\left\{\sup _{S_{k} \leq t \leq \Gamma_{k} \wedge T_{k+1}}|x(t)-2 k \pi| \leq \frac{(2+\delta) \eta_{\epsilon}}{\lambda_{+}-\lambda_{-}}\right\} \geq 1-e^{-C \epsilon^{-2 \zeta}}
$$

thus (6.27) follows from (6.39) and (6.40), whereas $(3.18)$ follows from $(6.38)$ and $(6.40) \cdot(3.16)$ and (3.17) descend both from (6.35) and (6.40).

\section{Conclusion of the Proofs}

We conclude the proofs of Propositions 3.7, 3.6 and 3.10 .

Proposition 6.10. Suppose that $\left(x_{k}, p_{k}\right) \in \mathcal{H}_{k}^{\xi}$ with $\xi$ small enough, then there exist $c^{\prime}>c>0$ such that

$$
c \frac{\eta_{\epsilon}^{(1+\theta)}}{\epsilon} \leq \frac{\left|P_{k}\left(S_{k}\right)-\wp_{k}^{*}\left(X_{k}\left(S_{k}\right)\right)\right|}{\sigma_{r}\left(T_{k}, S_{k}\right)\left|\wp_{k}(\bar{x})-\wp_{k}^{*}(\bar{x})\right|} \leq c^{\prime} \frac{\eta_{\epsilon}^{(1+\theta)}}{\epsilon}
$$

for any $\epsilon$ small enough.

Proof. Suppose that $\left(x_{k}, p_{k}\right) \in \mathcal{H}_{k}^{\xi}$. From 5.13 we know that

$$
\sigma_{r}^{2}\left(T_{k}, S_{k}\right)=\epsilon^{2} \Sigma_{r}^{(2)}\left[\wp_{k}, \bar{x}\right]\left(X_{k}\left(S_{k}\right)\right)
$$

where, by 4.27),

$$
\lim _{\epsilon \rightarrow 0} \frac{\Sigma_{r}^{(2)}\left[\wp_{k}, \bar{x}\right]\left(X_{k}\left(S_{k}\right)\right)}{\left[P_{k}\left(S_{k}\right)-\wp_{k}^{*}\left(X_{k}\left(S_{k}\right)\right)\right]^{2}}\left[\wp_{k}(\bar{x})-\wp_{k}^{*}(\bar{x})\right]^{2}(\bar{x}-2(k-1) \pi)^{2(1+\theta)} \in(0,+\infty)
$$

then, from (5.7) it follows that

$$
\lim _{\epsilon \rightarrow 0} \frac{\left|P_{k}\left(S_{k}\right)-\wp_{k}^{*}\left(X_{k}\left(S_{k}\right)\right)\right|}{\sigma_{r}\left(T_{k}, S_{k}\right)\left|\wp_{k}(\bar{x})-\wp_{k}^{*}(\bar{x})\right|} \frac{\epsilon}{\eta_{\epsilon}^{(1+\theta)}} \in(0,+\infty)
$$

from which the result. 
Lemma 6.11. There exist $C, c, c^{\prime}>0$ such that, for any $\zeta>0, \xi, \epsilon$ small enough,

$$
\mathbf{1}_{\left(x_{k}, p_{k}\right) \in \mathcal{H}_{k}^{\xi}} \mathbf{P}_{T_{k}, x_{k}, p_{k}}\left\{c \eta_{\epsilon}<2 k \pi-X_{k}\left(S_{k}\right) \leq c^{\prime} \eta_{\epsilon}\right\} \geq 1-e^{-C \epsilon^{-2 \zeta}}
$$

Proof. 6.45 follows from Lemma 6.6 and the monotonicity of $X_{k}(t)$.

Proof of Proposition 3.6. From Lemma 6.11 the hypothesis 4.3 holds for the couple $\bar{x}, X_{k}\left(S_{k}\right)$ with probability larger than $1-e^{-C \epsilon^{-2 \zeta}}$ then $(3.13$ directly follows from 6.42 and 4.44 .

Proof of Proposition 3.7. (3.14) easily follows from (6.41) and (5.9) since, from (2.19) and 2.20$)$ we have $\epsilon^{-1} \eta_{\epsilon}^{1+\theta} \bar{\sigma}_{\epsilon}=\epsilon^{\theta}$. (3.27) is a direct consequence of (3.14) and (3.13)

Let us define the processes

$$
Z_{k}(t)=\wp_{k}^{*}\left(X_{k}(t)\right)-\lambda_{-}\left(X_{k}(t)-2 k \pi\right), \quad V_{k}(t)=\wp_{k}^{*}\left(X_{k}(t)\right)-\lambda_{+}\left(X_{k}(t)-2(k-1) \pi\right)
$$

we have the following result.

Lemma 6.12. Let $\left(x_{k}, p_{k}\right) \in \mathcal{H}_{k}^{\xi}$ for some $\xi>0$, then there exists $c>0$ such that

$$
\left|V_{k}\left(T_{k}\right)\right| \leq c \eta_{\epsilon}^{2} \quad \mathbf{P}_{T_{k}, x_{k}, p_{k}} \text { a.s. }
$$

There exist $C, c^{\prime}>0$ such that, for any $\xi>0, \zeta, \epsilon$ small enough,

$$
\mathbf{1}_{\left(x_{k}, p_{k}\right) \in \mathcal{H}_{k}^{\xi}} \mathbf{P}_{T_{k}, x_{k}, p_{k}}\left\{\left|Z_{k}\left(S_{k}\right)\right| \leq c^{\prime} \eta_{\epsilon}^{2}\right\} \geq 1-e^{-C \epsilon^{-2 \zeta}}
$$

Proof. Recalling that $X_{k}\left(T_{k}\right)=x\left(T_{k}\right)=\bar{x}$, 6.46 follows from 4.1) and the left hand side of (5.7), whereas 6.47) follows from 4.2 and 6.45).

Lemma 6.13. There exist $C, c>0$ such that, for any $\zeta>0, \xi, \epsilon$ small enough,

$$
\mathbf{1}_{\left(x_{k}, p_{k}\right) \in \mathcal{H}_{k}^{\xi}} \mathbf{P}_{T_{k}, x_{k}, p_{k}}\left\{\left|\omega_{k}\left(S_{k}\right)-\lambda_{-}\right| \leq c \frac{\bar{\sigma}_{\epsilon}}{\eta_{\epsilon}}\right\} \geq 1-e^{-C \epsilon^{-2 \zeta}}
$$

Proof. We recall that $\omega_{k}\left(S_{k}\right)=\frac{d}{d x} \wp_{k}\left(X_{k}\left(S_{k}\right)\right)$. Suppose that $\left(x_{k}, p_{k}\right) \in \mathcal{H}_{k}^{\xi}, \xi$ small enough then, by Lemma 6.11 the hypothesis 4.3 holds for the couple $\bar{x}, X_{k}\left(S_{k}\right)$ with probability larger than $1-e^{-C \epsilon^{-2 \zeta}}$. Hence we can apply (4.23) and (5.9) and obtain that there exist $c, C>0$ such that for any $\zeta, \epsilon$ small enough,

$$
\mathbf{1}_{\left(x_{k}, p_{k}\right) \in \mathcal{H}_{k}^{\xi}} \mathbf{P}_{T_{k}, x_{k}, p_{k}}\left\{\left|\omega_{k}\left(S_{k}\right)-\frac{d}{d x} \wp_{k}^{*}\left(X_{k}\left(S_{k}\right)\right)\right| \leq c \frac{\bar{\sigma}_{\epsilon}}{\eta_{\epsilon}} \epsilon^{-\xi}\right\} \geq 1-e^{-C \epsilon^{-2 \zeta}}
$$


on the other hand, by 4.2 , there exist $c^{\prime}, C>0$ such that, for any $\zeta, \epsilon$ small enough,

$$
\mathbf{1}_{\left(x_{k}, p_{k}\right) \in \mathcal{H}_{k}^{\xi}} \mathbf{P}_{T_{k}, x_{k}, p_{k}}\left\{\left|\frac{d}{d x} \wp_{k}^{*}\left(X_{k}\left(S_{k}\right)\right)-\lambda_{-}\right| \leq c^{\prime} \eta_{\epsilon}\right\} \geq 1-e^{-C \epsilon^{-2 \zeta}}
$$

then 6.48 follows from 6.49) and 6.50 since $\eta_{\epsilon}^{2}=o\left(\bar{\sigma}_{\epsilon}\right)$.

Proof of Proposition 3.10. Suppose that $\left(x_{k}, p_{k}\right) \in \mathcal{H}_{k}^{\xi}$ for some $\xi>0$, then, from the definitions of $z_{k}(t)$ in (1.14), $\hat{z}_{k}(t)$ in (3.3) and $r_{k}(t)$ in (2.11) we have

$$
\begin{aligned}
z_{k}\left(S_{k}\right)-\hat{z}_{k}\left(S_{k}\right) & =Z_{k}\left(S_{k}\right)+\lambda_{-} q_{k}\left(S_{k}\right)-\omega_{k}\left(S_{k}\right) y_{k}\left(S_{k}\right) \\
& =Z_{k}\left(S_{k}\right)+r_{k}\left(S_{k}\right)+\left(\omega_{k}\left(S_{k}\right)-\lambda_{-}\right) y_{k}\left(S_{k}\right) \\
& =\mathcal{R}_{k}\left(S_{k}\right)+Z_{k}\left(S_{k}\right)+\left(\omega_{k}\left(S_{k}\right)-\lambda_{-}\right) y_{k}\left(S_{k}\right)
\end{aligned}
$$

where the last equivalence follows since $\bar{r}_{k}\left(S_{k}\right)=0$. Hence (3.19) descends from 6.1), 6.9), 6.47) and Lemma 6.13.

Suppose now that $\left(\bar{x}_{k-1}, \bar{p}_{k-1}\right) \in \mathcal{K}_{k-1}^{\xi}$ for some $\xi>0$. From the definitions of $v_{k}(t)$ in (1.14) and $\mathcal{V}_{k}(t)$ in $(2.4)$ we have

$$
\begin{aligned}
p\left(T_{k}\right)-\wp_{k}^{*}\left(x\left(T_{k}\right)\right)-\bar{v}_{k-1}\left(T_{k}\right) & =\mathcal{V}_{k-1}\left(T_{k}\right)-V_{k}\left(T_{k}\right)+\lambda_{+} y_{k}\left(T_{k}\right)+\wp_{k}^{*}\left(X_{k}\left(T_{k}\right)\right)-\wp_{k}^{*}\left(x\left(T_{k}\right)\right) \\
& =\mathcal{V}_{k-1}\left(T_{k}\right)-V_{k}\left(T_{k}\right)
\end{aligned}
$$

where the last equality follows since $X_{k}\left(T_{k}\right)=x\left(T_{k}\right)=x_{k}$. Then (3.20) descends from (3.17) and (6.46).

\section{Appendix}

In the present Appendix we provide a Gaussian Inequality and a comparison result.

Marcus-Shepp inequality for Gaussian processes. There is a classical result of Landau and Shepp [6] and Marcus and Shepp 7 that gives an estimate for the supremum of a general centered Gaussian process. If $X(t)$ is an a.s. bounded, centered Gaussian process of variance $\sigma^{2}(t)$, then,

$$
\lim _{\lambda \rightarrow \infty} \frac{1}{\lambda^{2}} \ln \mathbf{P}\left\{\sup _{t \in I} X(t) \geq \lambda\right\}=-\frac{1}{2 \sigma_{I}^{2}} \quad \text { with } \quad \sigma_{I}^{2}:=\sup _{t \in I} \sigma^{2}(t)
$$

An immediate consequence of (7.1) is that for any $\lambda$ large enough, $\delta$ small enough,

$$
\mathbf{P}\left\{\sup _{t \in I} X(t) \geq \lambda\right\} \leq e^{-\frac{\lambda^{2}}{2 \sigma_{I}^{2}}(1-\delta)}
$$

moreover, by symmetry we have

$$
\mathbf{P}\left\{\sup _{t \in I}|X(t)| \geq \lambda\right\} \leq 2 \mathbf{P}\left\{\sup _{t \in I} X(t) \geq \lambda\right\} .
$$

By applying the result to the process $X(t) / \sigma(t)$ we get

$$
\mathbf{P}\left\{\sup _{t} \frac{|X(t)|}{\sigma(t)} \geq \lambda\right\} \leq 2 e^{-\frac{\lambda^{2}}{2}(1-\delta)}
$$

for $\lambda$ large enough, $\delta$ small enough. 
Comparison with Gaussian Processes. In our proofs we repeatedly make use of a comparison argument comparing the solution of a linear SDE with the solution of a more general SDE, let us see. Let $X_{t}$ be a solution of the problem

$$
d X_{t}=\left(a(t) X_{t}+b(t)\right) d t+\xi d w_{t},
$$

with $a, b: \mathbb{R}^{+} \rightarrow \mathbb{R}$ bounded on bounded intervals and $\xi \in \mathbb{R}$, then $X(t)$ is a Gaussian process of the form

$$
X(t)=X\left(t_{0}\right) e^{\int_{t_{0}}^{t} a(s) d s}+\int_{t_{0}}^{t} b(s) e^{\int_{s}^{t} a\left(s^{\prime}\right) d s^{\prime}} d s+\xi \int_{t_{0}}^{t} e^{\int_{s}^{t} a\left(s^{\prime}\right) d s^{\prime}} d w_{s} .
$$

Consider, now, the processes $x(t)$ solution of

$$
d x_{t}=c\left(x_{t}, t\right) d t+\xi d w_{t}
$$

with the same noise of $(7.5), c: \mathbb{R} \times \mathbb{R}^{+} \rightarrow \mathbb{R}$ globally Lipschitz.

Lemma 7.1. For $X(t), x(t)$ as above we define $\delta_{t}:=c\left(X_{t}, t\right)-\left[a(t) X_{t}+b(t)\right], \Delta_{t}:=X_{t}-x_{t}$, and let $\tau \in \mathbb{R}^{+}$be a generic random variable. Suppose

$$
\operatorname{sign}\left(\Delta_{\tau}\right)=\operatorname{sign}\left(\delta_{\tau}\right) \quad \text { or } \quad \Delta_{\tau}=0,
$$

then

$$
\operatorname{sign}\left(\Delta_{t}\right)=\operatorname{sign}\left(\delta_{t}\right) \quad \text { for any } \quad \tau \leq t \leq \inf \left\{s \geq \tau: \delta_{s}=0\right\} \quad \text { a.s. }
$$

Proof. We have

$$
d \Delta_{t}=\left(a(t) \Delta_{t}+\delta_{t}\right) d t
$$

thus, for any $\tau \geq 0$

$$
\Delta(t)=\Delta(\tau) e^{\int_{\tau}^{t} a(s) d s}+\int_{\tau}^{t} \delta(s) e^{\int_{s}^{t} a\left(s^{\prime}\right) d s^{\prime}} d s
$$

then follows the result.

\section{References}

[1] Berglund N., Gentz B., Pathwise description of dynamic pitchfork bifurcations with additive noise, Prob. Theory Relat. Fields 122, 2002, 341-388

[3] Freidlin M. I., Wentzel A.D., Random Perturbations of Dynamical Systems SpringerVerlag 1984

[4] Hairer M. and Pavliotis G.A. From ballistic to diffusive motion in periodic potentials. J. Stat. Phys., 131 (1): 175-202, 2008.

[5] Kramers H.A., Brownian motion in a field of force and the diffusion model of chemical reactions Physica 7284 (1940)

[6] Landau H., Shepp L. A., On the Supremum of a Gaussian Process, Sankhya A, 32, 1970, 369-378

[7] Marcus M. B., Shepp L. A. Sample behaviour of Gaussian Processes, Proceedings of the 6th Berkeley Symposium on Mathematics, Stasistic and Probability, Vol.2, University of California Press, Berkeley, CA, 1972, 423-442. 
[8] Pavliotis G.A., Vogiannou A., Diffusive transport in periodic potentials: Underdamped dynamics, Fluct. and noise lett., 8, L155-L173, 2008

[9] Perez-Mato J.M., Blaha P., Schwarz K., Aroyo M., Orobengoa D., Etxebarria I., Garcia A., Multiple instabilities in $\mathrm{Bi}_{4} \mathrm{Ti}_{3} \mathrm{O}_{12}$ : A ferroelectric beyond the soft-mode paradigm Phys.Rev. B 77, 2008, 184104

[10] Perez-Mato J. M., Ribeiro J. L., Petricek V., Aroyo M. I., Magnetic superspace groups and symmetry constraints in incommensurate magnetic phases, J. Phys.: Condens. Matter 24, 2012, 163201

[11] Risken H. The Fokker-Planck Equation. Methods of Solution and Applications New York: Springer 1996

[12] Risken H., Vollmer H.D. Brownian Motion in Periodic Potentials in the Low-FrictionLimit; Nonlinear Response to an External Force Z. Physik B 35, 1979, 177-184.

[13] Stratonovich R. L. Topics in the theory of random noise. Vol. I. Revised English edition. Translated from the Russian by Richard A. Silverman. Gordon and Breach Science Publishers, New York, 1963.

[14] Stratonovich R. L. Topics in the theory of random noise. Vol. II. Revised English edition. Translated from the Russian by Richard A. Silverman. Gordon and Breach Science Publishers, New York, 1967. 